\title{
Nonlinear Control of an Active Heave Compensation System
}

\author{
K. D. Do* and J. Pan \\ School of Mechanical Engineering, The University of Western Australia \\ 35 Stirling Highway, Crawley, WA 6009, Australia \\ ${ }^{*}$ Corresponding author. Tel: +61864883125, Fax: +61864881024, \\ Email: duc@mech.uwa.edu.au (K. D. Do)
}

\begin{abstract}
Heave motion of a vessel or a rig has an adverse impact on the response of a drill-string or a riser. To compensate for heave motion, passive and active devices are usually used. Active heave compensators, in which a control system is an essential part, allow conducting operations under more extreme weather

ljkhij conditions than passive ones. This paper presents a constructive method to design a nonlinear controller for an active heave compensation system using an electro-hydraulic system driven by a double rod actuator. The control system reduces the effect of the heave motion of the vessel on the response of the riser by regulating the distance from the upper end of the riser to the seabed. The control development is based on Lyapunov's direct method and disturbance observers. The paper also includes a method to select the control and disturbance observer gains such that actuator saturations are avoided. Stability of the closed loop system is carefully examined. Simulation results illustrate the effectiveness of the proposed control system.
\end{abstract}

Keywords: Heave compensation, Nonlinear control, Riser.

\section{Introduction}

Offshore oilfield development has moved to a deeper, and more severe environment for new oil sources. Moreover, offshore oil-rigs have become small and light weight. Therefore, waves, wind and ocean currents easily cause the rig to move away from their desired position both horizontally and vertically. This in turn has adverse effects on the riser(s) connecting between the rig and the well at the sea bed, (Sarpkaya, 1981). The horizontal motion of the rig (hence the upper end of the risers) is often controlled by a dynamic positioning system in deep-water applications. Based on linear and nonlinear control theories, a number of control techniques for dynamic positioning systems has been proposed in literature, see for example (Grimble et al., 1980), (Fossen and

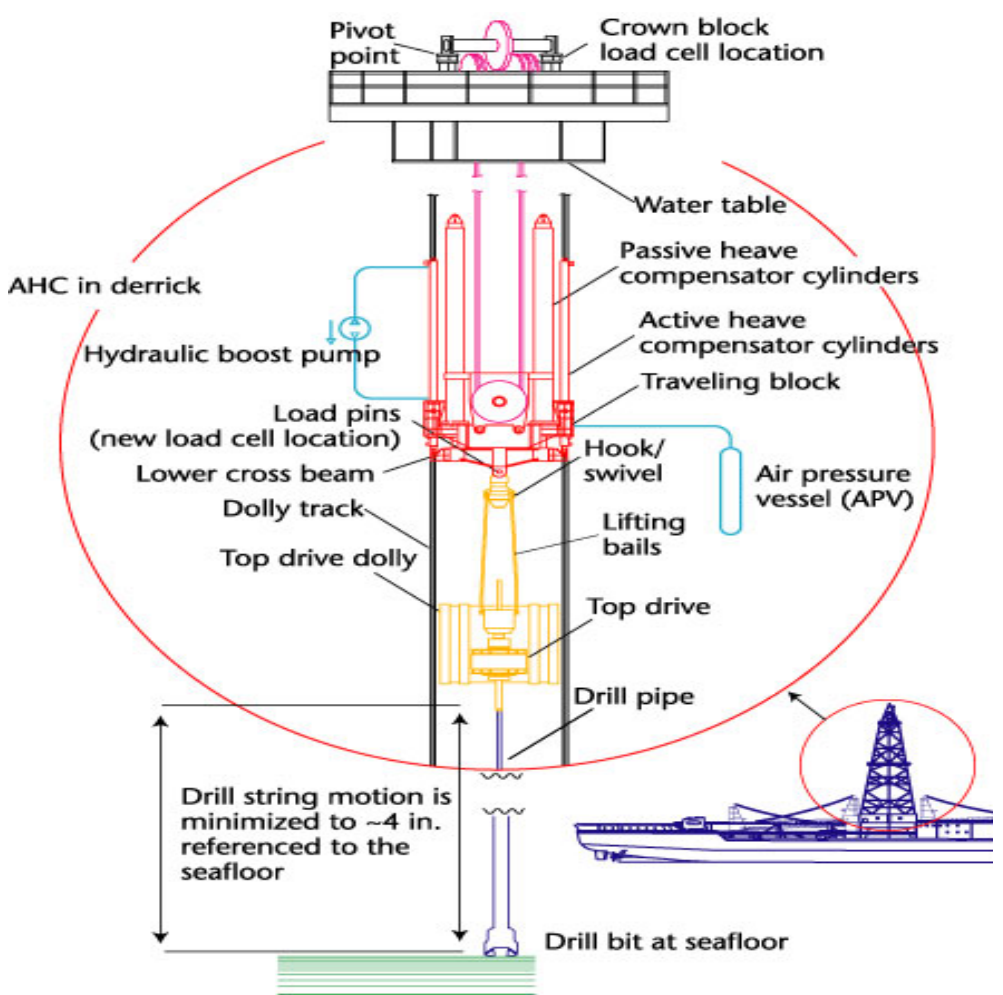

Figure 1: Schematic of an active heave compensation system (http://www.oceandrilling.org).

1 
Strand, 2001), (Do et al., 2002), (Soresen, 2005). However, in these papers the vertical (heave) motion of the rig, which directly changes the tension in the riser, is completely ignored. To reduce the effect of the heave motion of the rig, an active heave compensation system is often used in combination with a passive riser compensator to provide a stable position of the crown block referred to seabed. This system is based on a hydraulic cylinder installed on top of the passive crown block compensator. The cylinder is suspended by specially designed spherical bearings. The cylinder is operated in such a way that it applies force to the crown block to overcome frictions and the force acting between the riser and the active heave compensation unit to maintain a constant distance from the upper end of the riser to the seabed. The result is that the crown block position with respect to seabed is constant within $(0.1-0.5) \mathrm{m}$ with rig heave up to $(4-5) \mathrm{m}$, (Aker, 1998). The cylinder is controlled by means of input from a heave motion sensor (e.g. 3 axis accelerometer) and from a cylinder position sensor. A schematic of an active heave compensation system is depicted in Figure 1. Acting cylinders are attached directly between the crown block and the structure of the derrick.

It is surprising that while controlling dynamic positioning systems receives a lot of attention, control of active heave compensation systems has received much less attention from researchers. In (Korde, 1998), a control system based on linear control techniques is proposed for an active heave compensation system on drill-ships. Recently, active control of heave compensated cranes or module handling systems during the water entry phase of a subsea installation was studied in (Johansen et al., 2003). In this work, a concept referred to as wave synchronization is introduced, where the main idea is to utilize a wave amplitude measurement in order to compensate directly for the water motion due to waves inside the moonpool. In (Skaare and Egeland, 2006), the authors proposed a parallel force/position controller for the control of loads through the wave zone in marine operations. The controller structure has similarities to the parallel force/position control scheme used in robotics. Their controller achieved a significant improvement of the minimum value of the wire tension over the wave synchronization approach which was used in (Johansen et al., 2003). In (Marconi et al., 2002), an autopilot for the autonomous landing of a vertical take off and landing vehicle on a ship whose deck oscillates in the vertical direction due to high sea states is proposed. The heave motion of the deck is compensated using an internalmodel-based error feedback dynamic regulator. Designing a high performance controller for an active heave compensation system is a challenging task. This is partially due to the fact that the force acting between the riser/drill-string and the active heave compensation unit is difficult to model accurately. Moreover, due to complex response of the riser/drill-string it is complicated to determine the upper bound of this force as a function of the hydraulic piston motion. This upper bound is usually required in robust and adaptive control design methods, see (Krstic et al., 1995) and (Khalil, 2002) for example.

This paper focuses on an active heave compensation objective, which is defined as minimization of the effect of the heave motion of the vessel on the response of the riser by regulating the distance from the upper end of the riser to the seabed. We present a constructive method to design a nonlinear controller for an active heave compensation system using an electro-hydraulic system driven by a double rod actuator. A disturbance observer is also developed to estimate the force acting on the piston of the hydraulic system, and the heave acceleration of the vessel. This disturbance observer is then implemented in the control design to result in a high performance control system. The control development and stability analysis are based on Lyapunov's direct method. 


\section{Problem formulation and preliminaries}

\subsection{Problem formulation}

We consider an active heave compensation system depicted in Figure 2. It consists of an electrohydraulic system driven by a double rod actuator. The riser connects to the piston of the hydraulic system via a ball joint. Hence there is no bending moment. The hydraulic system's house is fixed to the vessel/rig. The heave motion of the vessel/rig is denoted by $z(t)$, which is coordinated in the earth-fixed frame, $O_{E} X_{E} Y_{E} Z_{E}$. Let $x_{H}$, which is coordinated in the vessel body-fixed frame, be the displacement of the cylinder rod (piston) of the hydraulic system, i.e. the motion of the piston with respect to the vessel in the vertical direction. The mathematical formulation and control design in this paper is generic. Hence they also work for one or more hydraulic systems.

To regulate the distance from the upper end of the riser to the seabed, it is obvious that we want to keep the sum $\left|x_{H}(t)+z(t)-L\right|$ as small as possible, where $L$ is a constant, which is preset to achieve the desired pretension of the riser. This is done in the calibration process when the riser system is installed. Let $i_{H}$ be the current input to the hydraulic system. Then the control objective can be described as designing the control input $i_{H}$ to drive the cylinder rod in such a way that $\left|x_{H}(t)+z(t)-L\right|$ is kept as small as possible. In sequel, we will develop a mathematical model describing dynamics of the active heave compensation system to formulate the control objective. The dynamics of the piston/rod can be described as

$$
\begin{aligned}
m_{H} \ddot{x}_{H}= & A_{H} P_{H}-b_{H} \dot{x}_{H} \\
& +\tilde{\Delta}\left(t, x_{H}, z, \dot{x}_{H}, \dot{z}\right)
\end{aligned}
$$

where $m_{H}$ is the mass of the rod of the hydraulic system, $P_{H}=P_{1}-P_{2}$ is the load pressure of the cylinder with $P_{1}$ and $P_{2}$ being the pressures in the upper and lower compartments of the

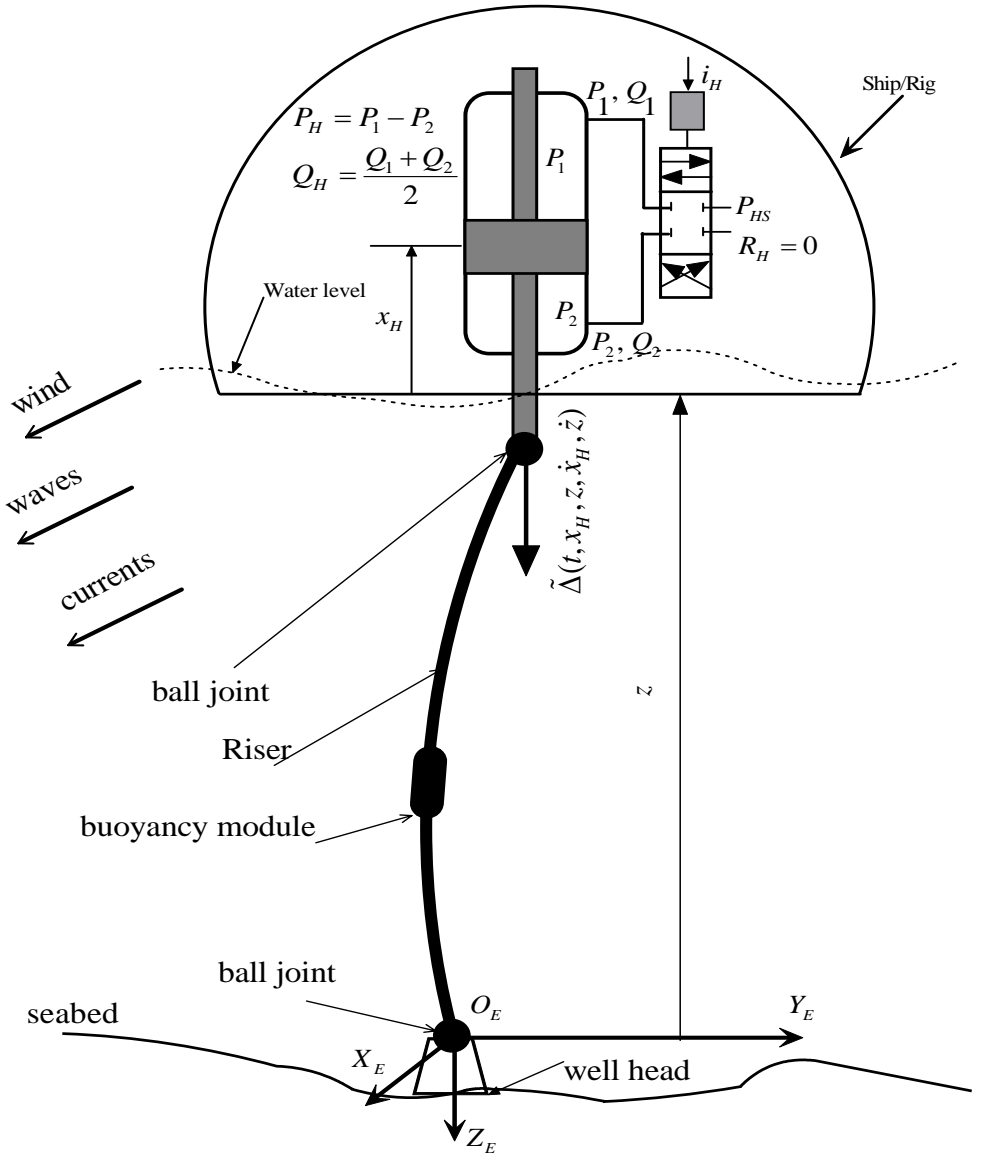

Figure 2: Representative structure of an active heave compensation system cylinder, see Figure 2, $A_{H}$ is the ram area of the cylinder, $b_{H}$ represents the combined coefficient of the modeled damping and viscous friction forces on the cylinder rod, and $\tilde{\Delta}\left(t, x_{H}, z, \dot{x}_{H}, \dot{z}\right)$ denotes the force acting on the rod from the riser or drill-string. It is noted that there would be a force from the riser acting on the cylinder rod in the horizontal plane. This force will affect the motions in sway and surge. However, we do not consider the sway and surge motions, which are considered in a dynamic positioning system, in this paper. It is complicated to derive 
an explicit formula for the force $\tilde{\Delta}\left(t, x_{H}, z, \dot{x}_{H}, \dot{z}\right)$. This force would depend on many factors such as $x_{H}, z, \dot{x}_{H}, \dot{z}$, initial tension in the riser, weight of the riser, current profile and current magnitude acting on the riser, and shape of the riser. In addition, the term $\tilde{\Delta}\left(t, x_{H}, z, \dot{x}_{H}, \dot{z}\right)$ can include unmodeled friction force between the piston and the cylinder of the hydraulic system. This friction force is also difficult to accurately model. Hence, in this paper we consider the force $\tilde{\Delta}\left(t, x_{H}, z, \dot{x}_{H}, \dot{z}\right)$ as a disturbance. We do not ignore this disturbance but will later develop a disturbance observer to estimate $\tilde{\Delta}\left(t, x_{H}, z, \dot{x}_{H}, \dot{z}\right)$ for the control design purpose. Neglecting the leakage flows in the cylinder and the servovalve, the actuator or the cylinder dynamics is written as (Merritt, 1967)

$$
\frac{V_{H}}{4 \beta_{H e}} \dot{P}_{H}=-A_{H} \dot{x}_{H}-C_{H T} P_{H}+Q_{H}
$$

where $V_{H}$ is the total volume of the cylinder and the hoses between the cylinder and the servovalve, $\beta_{H e}$ is the effective bulk modulus, $C_{H T}$ is the coefficient of the total internal leakage of the cylinder due to pressure, $Q_{H}$ is the load flow. The load flow $Q_{H}$ is related to the spool displacement of the servovalve, $x_{H v}$, by (Merritt, 1967)

$$
Q_{H}=C_{H D} W_{H} x_{H v} \sqrt{\frac{P_{H S}-\operatorname{sgn}\left(x_{H v}\right) P_{H}}{\rho_{H}}}
$$

where $C_{H D}$ is the discharge coefficient, $W_{H}$ is the spool valve area gradient, $P_{H S}$ is the supply pressure of the fluid, sgn denotes the standard signum function, and $\rho_{H}$ is density of the oil. It is noted that since the supply pressure $P_{H S}$ is always higher than the load pressure $P_{H}$, i.e. there exists a strictly positive constant $\epsilon_{1}$ such that $P_{H S}-\operatorname{sgn}\left(x_{H v}\right) P_{H} \geq \epsilon_{1}$. Hence the equation (3) is well-defined all $x_{H v} \in \mathbb{R}$. The servovalve dynamics can be described as

$$
\tau_{H v} \dot{x}_{H v}=-x_{H v}+k_{H v} i_{H}
$$

where $\tau_{H v}$ and $k_{H v}$ are the time constant and gain of the servovalve, respectively, $i_{H}$ is the current input to the hydraulic system. Since $P_{H}$ is usually very large and $\tau_{H v}$ is usually very small, we scale the pressure $P_{H}$ and the spool displacement $x_{H v}$ as $\bar{P}_{H}=\frac{P_{H}}{C_{H 3}}$ and $\bar{x}_{H v}=\frac{x_{H v}}{C_{H 4}}$ where $C_{H 3}$ and $C_{H 4}$ are constants, to avoid numerical error and facilitating the control gain tuning process. Now the entire system consisting of (11), (2) and (44) can be rewritten as

$$
\begin{aligned}
\ddot{x}_{H} & =\frac{A_{H} C_{H 3}}{m_{H}} \bar{P}_{H}-\frac{b_{H}}{m_{H}} \dot{x}_{H}+\frac{1}{m_{H}} \tilde{\Delta}\left(t, x_{H}, z, \dot{x}_{H}, \dot{z}\right), \\
\dot{\bar{P}}_{H} & =-\frac{4 \beta_{H e} A_{H}}{V_{H} C_{H 3}} \dot{x}_{H}-\frac{4 \beta_{H e} C_{H T}}{V_{H}} \bar{P}_{H}+\frac{4 \beta_{H e} C_{H D} W_{H} C_{H 4} \bar{x}_{H v}}{V_{H} \sqrt{\rho_{H}} \sqrt{C_{H 3}}} \sqrt{\bar{P}_{H S}-\operatorname{sgn}\left(\bar{x}_{H v}\right) \bar{P}_{H}}, \\
\dot{\bar{x}}_{H v} & =-\frac{1}{\tau_{H v}} \bar{x}_{H v}+\frac{k_{H v}}{\tau_{H v} C_{H 4}} i_{H}
\end{aligned}
$$

where $\bar{P}_{H S}=\frac{P_{H S}}{C_{H 3}}$. For stabilization/tracking control of the hydraulic system (5) without using disturbance observers but the "concept of dominating the size of disturbances", the reader is referred to (Yao et al., 2001) and references therein. We now restate the control objective as follows:

Control objective. Under the assumption that $z(t)$ and $\tilde{\Delta}\left(t, x_{H}, z, \dot{x}_{H}, \dot{z}\right)$ and their derivatives are bounded, design the control input $i_{H}$ to regulate the distance from the upper end of the riser to seabed, i.e. to keep the sum $\left|z(t)+x_{H}(t)-L\right|$ as small as possible, where $L$ is a 
constant which is preset to achieve a desired tension in the riser.

Remark 1. A heave compensation system is usually calibrated in such a way that at the zero vessel/rig heave, the riser tension is at its desired value, i.e. the constant $L$ is available for the control design. Since the dynamics of the vessel/rig and the riser in large motion mode are slow (i.e. we do not consider vibration mode of the riser in this paper), we can assume boundedness of $z(t)$ and $\tilde{\Delta}\left(t, x_{H}, z, \dot{x}_{H}, \dot{z}\right)$ and their derivatives in the control design. Therefore, assumptions made in the above control objective are reasonable in practice.

\subsection{Preliminaries}

Since we assume that $\tilde{\Delta}\left(t, x_{H}, z, \dot{x}_{H}, \dot{z}\right)$ and $\dot{z}(t)$ are not available for the control design, we present in this subsection a disturbance observer, which will be used to estimate $\tilde{\Delta}\left(t, x_{H}, z, \dot{x}_{H}, \dot{z}\right)$ and $\dot{z}(t)$ in the control design in the next section. In addition, discontinunity of the term $\sqrt{\bar{P}_{H S}-\operatorname{sgn}\left(\bar{x}_{H v}\right) \bar{P}_{H}}$ in (5) causes difficulty when taking derivatives of this term with respect to time in the control design. Therefore, we also present a $p$-times differentiable signum function to approximate the signum function in (15).

\subsubsection{Disturbance observer}

Consider the following system

$$
\dot{x}=f(x)+u+d(t, x)
$$

where $x \in \mathbb{R}^{n}, f(x)$ is a vector of known functions of $x, u$ is the control input vector, and $d(t, x)$ is a vector of unknown disturbances. We assume that there exists a nonnegative constant $C_{d}$ such that $\|\dot{d}(t, x)\| \leq C_{d}$. Now we want to design the control input $u$ to stabilize the system (6) at the origin. It is obvious that if we can design a disturbance observer, $\hat{d}(t, x)$, that estimates $d(t, x)$ sufficiently accurately, then the control input $u$ is straightforwardly designed as $u=-\kappa x-f(x)-\hat{d}(t, x)$ with $\kappa$ a positive definite matrix. The disturbance observer is given in the following lemma.

Lemma 1. Consider the following disturbance observer

$$
\begin{aligned}
& \hat{d}(t, x)=\xi+\rho(x) \\
& \dot{\xi}=-K(x) \xi-K(x)(f(x)+u+\rho(x))
\end{aligned}
$$

where $K(x)=\frac{\partial \rho(x)}{\partial x}, \rho(x)$ is chosen such that the matrix $K(x)$ is positive definite for all $x \in \mathbb{R}^{n}$. The disturbance observer (7) guarantees that the disturbance observer error $d_{e}(t, x)=$ $d(t, x)-\hat{d}(t, x)$ exponentially converges to a ball centered at the origin. The radius of this ball can be made arbitrarily small by adjusting the function $\rho(x)$. In the case $C_{d}=0$, the disturbance observer error $d_{e}(t, x)$ exponentially converges to zero.

Proof. See Appendix A. The disturbance observer (7) is a dynamical system. The variable $\xi$ is generated by the second equation of (7), which is an ordinary differential equation, with some initial value $\xi\left(t_{0}\right)$, where $t_{0}$ is the initial time. The choice of the function $\rho(x)$, which results in the matrix $K(x)$ directly affects performance of the disturbance observer. The larger eigenvalues of the matrix $K(x)$ are the faster the response of the disturbance observer is with a trade-off of a large overshoot of the observer, and vice-versa. The initial value $\xi\left(t_{0}\right)$ is ideally chosen to be 
$d\left(t_{0}, x\left(t_{0}\right)\right)-\rho\left(x\left(t_{0}\right)\right)$, where $d\left(t_{0}, x\left(t_{0}\right)\right)$ and $\rho\left(x\left(t_{0}\right)\right)$ are the initial values of $d(t, x)$ and $\rho(x)$, respectively. However, $d\left(t_{0}, x\left(t_{0}\right)\right)$ is unavailable since the disturbance $d(t, x)$ is unknown, we usually take $\xi\left(t_{0}\right)=0$ in practice. Of course, one can choose $\xi\left(t_{0}\right)$ to be any constant but the transient response of the disturbance observer might be bad because as $d\left(t_{0}, x\left(t_{0}\right)\right)$ is unknown, the chosen constant $\xi\left(t_{0}\right)$ can make $\left|d\left(t_{0}, x\left(t_{0}\right)\right)-\rho\left(x\left(t_{0}\right)\right)\right|$ large.

To illustrate the effectiveness of the disturbance observer (7), we perform some numerical simulations. In the simulations, we consider a scalar system in the form of (6) with $f(x)=$ $\arctan \left(x+x^{2}\right)$ and $d(t, x)=\sum_{i=1}^{5}\left(\sin (i t)+\sin (x) \sin \left(\frac{i t}{2}\right)\right)$. The function $\rho(x)$ is taken as $\rho(x)=20\left(x+\frac{x^{3}}{3}\right)$. This choice gives $K(x)=20\left(1+x^{2}\right)$, which is positive for all $x \in \mathbb{R}$. The initial conditions are $x(0)=1$ and $\xi(0)=0$. The control law is designed as $u=-\kappa x-f(x)-\hat{d}(t, x)$ with $\kappa=5$. We run two simulations. In the first one, the disturbance $d(t, x)$ is ignored in the control design, i.e. we set $\hat{d}(t, x)=0$ in the above control law. Simulation results are presented in the top two sub-figures A) and B) of Figure 3 . In the second simulation, we include $\hat{d}(t, x)$ in the control law. Simulation results are plotted in the bottom two sub-figures C) and D) of Figure 3, It is seen from the sub-figures A) and C) that in the case where the disturbance $d(t, x)$ is ignored in the control design, the state $x$ converges to a much larger ball than the case where the disturbance observer is used in the control design. In the sub-figures B) and D), the disturbance $d(t, x)$ is plotted in the solid line while the disturbance estimate $\hat{d}(t, x)$ is plotted in the dash-dotted line. From the sub-figure D), we can see that the disturbance observer provides an excellent estimate of the time and state dependent disturbance $d(t, x)$. Finally, it is noted that the value of disturbance $d(t, x)$ plotted in the sub-figure $\mathrm{B})$ is slightly different from that of the one plotted in the sub-figure $\mathrm{D}$ ) because the disturbance $d(t, x)$ depends on the state $x$.
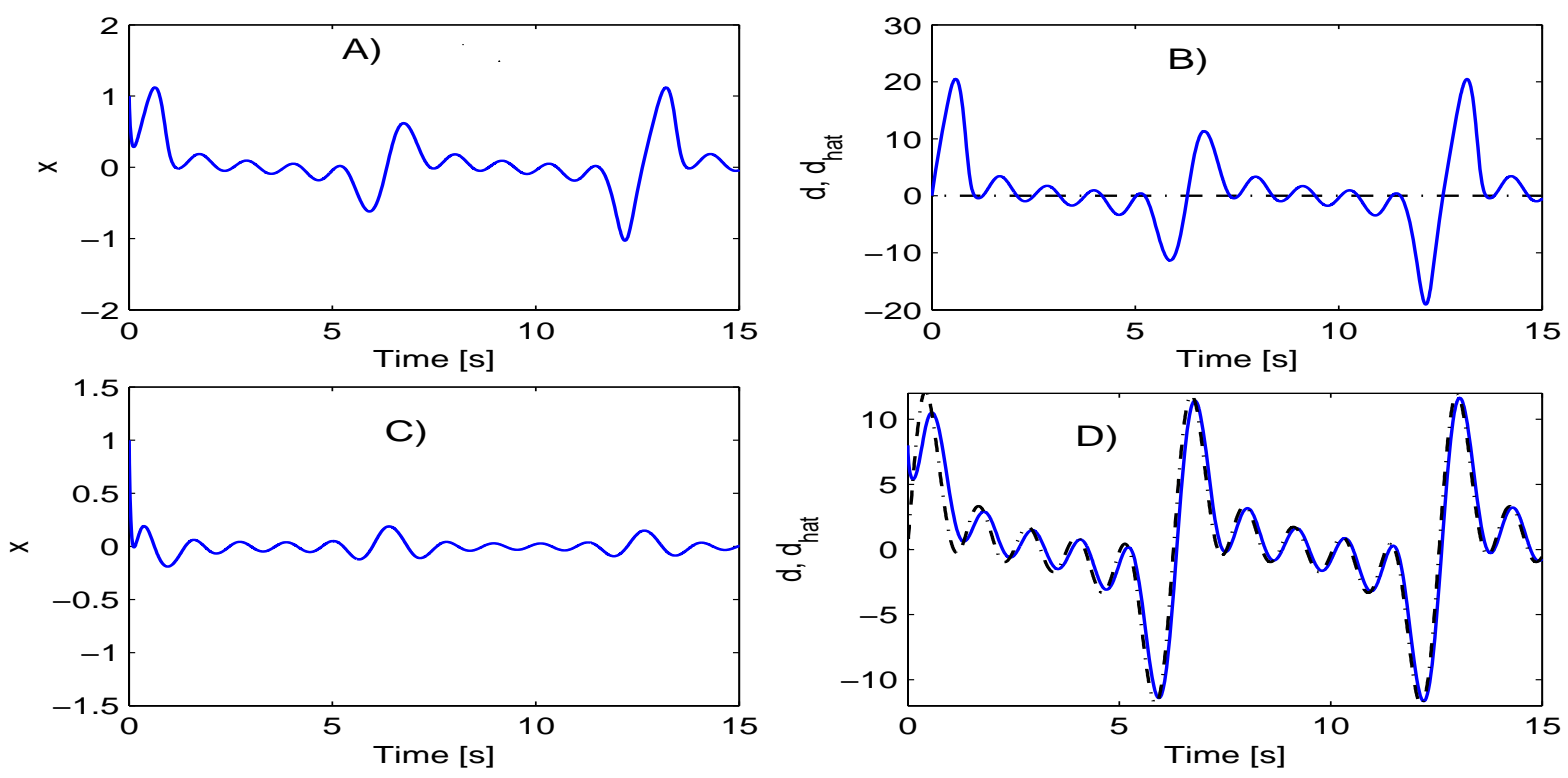

Figure 3: Effectiveness of the proposed disturbance observer.

\subsection{2 $p$-times differentiable signum function}


Definition 1. A scalar function $h(x, a, b)$ is called a $p$-times differentiable signum function if it enjoys the following properties

1) $h(x, a, b)=-1 \quad$ if $\quad-\infty<x \leq a$,

2) $h(x, a, b)=1 \quad$ if $\quad x \geq b$,

3) $-1<h(x, a, b)<1$ if $a<x<b$,

4) $h(x, a, b)$ is $p$ times differentiable

with respect to $x$

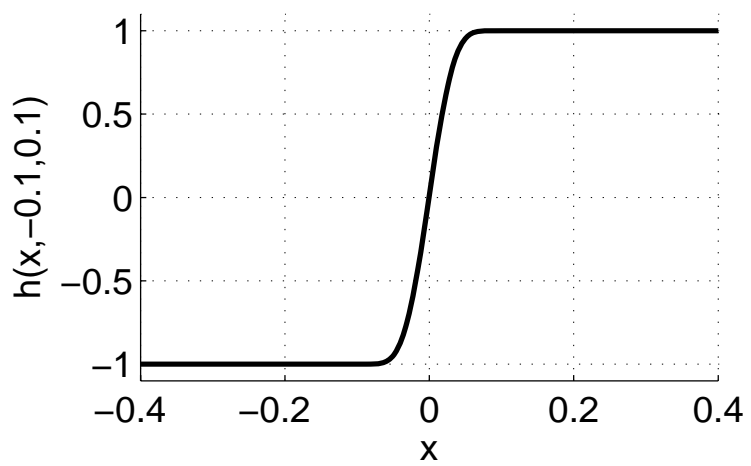

where $p$ is a positive integer, $x \in \mathbb{R}_{+}$, and $a$ Figure 4: A twice differentiable signum function. and $b$ are constants such that $a<0<b$. Moreover, if the function $h(x, a, b)$ is infinite times differentiable with respect to $x$, then it is called a smooth signum function.

Lemma 2. Let the scalar function $h(x, a, b)$ be defined as

$$
h(x, a, b)=2 \frac{\int_{a}^{x} f(\tau-a) f(b-\tau) d \tau}{\int_{a}^{b} f(\tau-a) f(b-\tau) d \tau}-1
$$

where the function $f(y)$ is defined as follows

$$
f(y)=0 \text { if } y \leq 0 \text { and } f(y)=y^{p} \text { if } y>0
$$

with $p$ being a positive integer. Then the function $h(x, a, b)$ is a $p$ times differentiable signum function. Moreover, if $f(y)=y^{p}$ in (10) is replaced by $f(y)=e^{-1 / y}$ then property 4) is replaced by $\left.4^{\prime}\right) h(x, a, b)$ is a smooth signum function.

Proof. See Appendix B. An illustration of a twice differentiable signum function $(a=$ $-0.1, b=0.1)$ is given in Fig. 4 .

Remark 2. As specified in Lemma 2, the $p$-times differentiable signum functions can be obtained explicitly, i.e. the integral (9) can be solved analytically. The smooth signum functions cannot obtained explicitly, i.e. the integral (9) cannot be solved analytically, thus a numerical procedure is needed to solve the integral (9). A simpler version of the $p$-times differentiable signum function was also given in (Do, 2007).

With materials presented in this subsection, we rewrite the entire system of the heave compensation dynamics (5) in a standard state space form for the purpose of control design in the next section as follows:

$$
\begin{aligned}
& \dot{x}_{1}=x_{2}, \\
& \dot{x}_{2}=\theta_{21} x_{3}-\theta_{22} x_{2}+\Delta\left(t, x_{1}, x_{2}, z, \dot{z}\right), \\
& \dot{x}_{3}=-\theta_{31} x_{2}-\theta_{32} x_{3}+\theta_{33} g_{3}\left(x_{3}, x_{4}\right), \\
& \dot{x}_{4}=-\theta_{41} x_{4}+\theta_{42} i_{H}
\end{aligned}
$$


with $x_{1}=x_{H}, x_{2}=\dot{x}_{H}, x_{3}=\bar{P}_{H}, x_{4}=\bar{x}_{H v}$, and

$$
\begin{aligned}
& \theta_{21}=\frac{A_{H} C_{H 3}}{m_{H}}, \theta_{22}=\frac{b_{H}}{m_{H}}, \Delta\left(t, x_{1}, x_{2}, z, \dot{z}\right)=\frac{1}{m_{H}} \tilde{\Delta}\left(t, x_{H}, z, \dot{x}_{H}, \dot{z}\right) \\
& \theta_{31}=\frac{4 \beta_{H e} A_{H}}{V_{H} C_{H 3}}, \theta_{32}=\frac{4 \beta_{H e} C_{H T}}{V_{H}}, \theta_{33}=\frac{4 \beta_{H e} C_{H D} W_{H} C_{H 4}}{V_{H} \sqrt{\rho_{H}} \sqrt{C_{H 3}}}, g_{3}\left(x_{3}, x_{4}\right)=x_{4} \sqrt{\bar{P}_{H S}-h\left(x_{4}, a, b\right) x_{3}}, \\
& \theta_{41}=\frac{1}{\tau_{H v}}, \theta_{42}=\frac{k_{H v}}{\tau_{H v} C_{H 4}}
\end{aligned}
$$

where we have used the $p$-times differentiable signum function $h\left(x_{4}, a, b\right)$ to replace the signum function $\operatorname{sgn}\left(x_{4}\right)$.

Remark 3. The use of the $p$-times differentiable signum function $h\left(x_{4}, a, b\right)$ instead of the signum function $\operatorname{sgn}\left(x_{4}\right)$ in (11) not only makes the the function $g_{3}\left(x_{3}, x_{4}\right)$ differentiable with respect to $x_{3}$ and $x_{4}$ but also represents the actual dynamics of the spool dynamics. This is because there is always certain inaccuracy in manufacturing the servovalve, i.e. the flow in the servovalve does not change its direction immediately.

\section{Control design}

A close look at the entire system (11) shows that the system is of a strick-feedback form, (Krstic et al., 1995). Therefore, we will use the backstepping technique to design the control input $i_{H}$ to achieve the control objective stated in the previous section. The control design consists of 4 steps. At steps 1 and 2, we will use the disturbance observer presented in Subsection 2.2.1 to design an estimate for $\dot{z}(t)$ and $\Delta\left(t, x_{1}, x_{2}, z, \dot{z}\right)$.

\subsection{Step 1}

Based on the backstepping technique, at this step we consider the first equation of the entire system (11) where the state $x_{2}$ is considered as a control. As such, we define

$$
\begin{aligned}
& x_{1 e}=x_{1}+z(t)-L, \\
& x_{2 e}=x_{2}-\alpha_{1}
\end{aligned}
$$

where $\alpha_{1}$ is a virtual control of $x_{2}$. Differentiating both sides of the first equation of (13) along the solutions of the second equation of (13) and the first equation of (11) gives

$$
\dot{x}_{1 e}=\alpha_{1}+x_{2 e}+w
$$

where $w:=\dot{z}(t)$. The goal is to design the virtual control $\alpha_{1}$ to stabilize $x_{1 e}$ at the origin while treating $w$ as a disturbance. Also at this step, we do not need to consider $x_{2 e}$. Regulating $x_{2 e}$ to a small neighborhood of the origin will be done at the next step. Ideally, we would regulate $x_{2 e}$ to the origin but it is impossible since (14) contains a time varying disturbance $w$ and the disturbance observer can only estimate the time varying disturbance $w$ approximately. It is seen that (14) is exactly the same form as (6) if we ignore $x_{2 e}$. Hence, from (14) and the disturbance observer design proposed in Subsection 2.2.1, we design the virtual control $\alpha_{1}$ and the estimate 
$\hat{w}$ of $w$ as follows:

$$
\begin{aligned}
& \alpha_{1}=-k_{11} x_{1 e}-\hat{w} \\
& \hat{w}=\xi_{1}+k_{12} x_{1 e} \\
& \dot{\xi}_{1}=-k_{12} \xi_{1}-k_{12}\left(x_{2 e}+\alpha_{1}+k_{12} x_{1 e}\right)
\end{aligned}
$$

where $k_{11}$ and $k_{12}$ are positive constants. It is noted that the above disturbance observer is an application from Subsection 2.2.1 where we took $\rho(x)=k_{12} x$. Using (14) and (15), we have the following first closed loop sub-system

$$
\begin{aligned}
& \dot{x}_{1 e}=-k_{11} x_{1 e}+x_{2 e}+w_{e}, \\
& \dot{w}_{e}=-k_{12} w_{e}+\dot{w}
\end{aligned}
$$

where $w_{e}=w-\hat{w}$. The dynamics (16) will be used in stability analysis of the closed loop system. It is of interest to note that the virtual control $\alpha_{1}$ is a smooth function of $x_{1 e}$ and $\xi_{1}$.

\section{$3.2 \quad$ Step 2}

Our goal at this step is to regulate $x_{2 e}$ to a small neighborhood of the origin by considering the second equation of the entire system (11). Similarly to Step 1, the state $x_{3}$ is considered as a control. As such, we define

$$
x_{3 e}=x_{3}-\alpha_{2}
$$

where $\alpha_{2}$ is a virtual control of $x_{3}$. Now differentiating both sides of the second equation of (13) along the solutions of the second equation of (11), (15) and (16) gives

$$
\dot{x}_{2 e}=\theta_{21}\left(x_{3 e}+\alpha_{2}\right)-\theta_{22}\left(x_{2 e}+\alpha_{1}\right)+\Delta\left(t, x_{1}, x_{2}, z, \dot{z}\right)-\frac{\partial \alpha_{1}}{\partial x_{1 e}}\left(-k_{11} x_{1 e}+x_{2 e}+w_{e}\right)-\frac{\partial \alpha_{1}}{\partial \xi_{1}} \dot{\xi}_{1} .
$$

Again at this step we do not consider $x_{3 e}$, of which regulation to a small neighborhood of the origin will be done at the next step. Clearly, it is seen that (18) is the same form as (6) in Subsection 2.2.1 if we ignore $x_{3 e}$. Therefore, from (18) and the disturbance observer design proposed in Subsection 2.2.1, we choose the virtual control $\alpha_{2}$ and an estimate $\hat{\Delta}$ of the disturbance $\Delta\left(t, x_{1}, x_{2}, z, \dot{z}\right)$ as follows

$$
\begin{aligned}
\alpha_{2} & =\frac{1}{\theta_{21}}\left(-x_{1 e}-k_{21} x_{2 e}+\theta_{22}\left(x_{2 e}+\alpha_{1}\right)+\frac{\partial \alpha_{1}}{\partial x_{1 e}}\left(-k_{11} x_{1 e}+x_{2 e}\right)+\frac{\partial \alpha_{1}}{\partial \xi_{1}} \dot{\xi}_{1}-\hat{\Delta}\right), \\
\hat{\Delta} & =\xi_{2}+k_{22} x_{2 e}, \\
\dot{\xi}_{2} & =-k_{22} \xi_{2}-k_{22}\left(\theta_{21}\left(x_{3 e}+\alpha_{2}\right)-\theta_{22}\left(x_{2 e}+\alpha_{1}\right)-\frac{\partial \alpha_{1}}{\partial x_{1 e}}\left(-k_{11} x_{1 e}+x_{2 e}\right)-\frac{\partial \alpha_{1}}{\partial \xi_{1}} \dot{\xi}_{1}\right)
\end{aligned}
$$

where $k_{21}$ and $k_{22}$ are positive constants. It is again noted that the above disturbance observer is an application from Subsection 2.2.1 where we took $\rho(x)=k_{22} x$. Using (18) and (19), we have the following second closed loop sub-system

$$
\begin{aligned}
& \dot{x}_{2 e}=-x_{1 e}-k_{21} x_{2 e}+\theta_{21} x_{3 e}-\frac{\partial \alpha_{1}}{\partial x_{1 e}} w_{e}+\Delta_{e}, \\
& \dot{\Delta}_{e}=-k_{22} \Delta_{e}+k_{22} \frac{\partial \alpha_{1}}{\partial x_{1 e}} w_{e}+\dot{\Delta}
\end{aligned}
$$

where $\Delta_{e}=\Delta-\hat{\Delta}$. The dynamics (20) will be used in stability analysis of the closed loop system. It is noted that the virtual control $\alpha_{2}$ is a smooth function of $x_{1 e}, \xi_{1}, x_{2 e}$ and $\xi_{2}$. 


\section{$3.3 \quad$ Step 3}

The objective of this step is to regulate $x_{3 e}$ to a small neighborhood of the origin by considering the third equation of the entire system (11). To simplify the design process, we consider $g_{3}\left(x_{3}, x_{4}\right)$ a control instead of $x_{4}$. As such, we define

$$
x_{4 e}=g_{3}\left(x_{3}, x_{4}\right)-\alpha_{3}
$$

where $\alpha_{3}$ is a virtual control of $g_{3}\left(x_{3}, x_{4}\right)$. Now differentiating both sides of (17) along the solutions of the third equation of (11), (15), (16) and (20) gives

$$
\begin{gathered}
\dot{x}_{3 e}=-\theta_{31} x_{2}-\theta_{32} x_{3}+\theta_{33}\left(\alpha_{3}+x_{4 e}\right)-\frac{\partial \alpha_{2}}{\partial x_{1 e}}\left(-k_{11} x_{1 e}+x_{2 e}+w_{e}\right)-\frac{\partial \alpha_{2}}{\partial \xi_{1}} \dot{\xi}_{1}- \\
\frac{\partial \alpha_{2}}{\partial x_{2 e}}\left(-x_{1 e}-k_{21} x_{2 e}+\theta_{21} x_{3 e}-\frac{\partial \alpha_{1}}{\partial x_{1 e}} w_{e}+\Delta_{e}\right)-\frac{\partial \alpha_{2}}{\partial \xi_{2}} \dot{\xi}_{2} .
\end{gathered}
$$

Again at this step we do not consider $x_{4 e}$, of which regulation to a small neighborhood of the origin will be done at the next step. From (22), we choose the virtual control $\alpha_{3}$ as

$$
\begin{aligned}
& \alpha_{3}=\frac{1}{\theta_{33}}\left(-\theta_{21} x_{2 e}-k_{31} x_{3 e}+\theta_{31} x_{2}+\theta_{32} x_{3}+\frac{\partial \alpha_{2}}{\partial x_{1 e}}\left(-k_{11} x_{1 e}+x_{2 e}\right)+\frac{\partial \alpha_{2}}{\partial \xi_{1}} \dot{\xi}_{1}+\right. \\
& \left.\frac{\partial \alpha_{2}}{\partial x_{2 e}}\left(-x_{1 e}-k_{21} x_{2 e}+\theta_{21} x_{3 e}\right)+\frac{\partial \alpha_{2}}{\partial \xi_{2}} \dot{\xi}_{2}\right)
\end{aligned}
$$

where $k_{31}$ is a positive constant. Now substituting (23) into (22) gives the following third closed loop sub-system

$$
\dot{x}_{3 e}=-\theta_{21} x_{2 e}-k_{31} x_{3 e}+\theta_{33} x_{4 e}-\left(\frac{\partial \alpha_{2}}{\partial x_{1 e}}-\frac{\partial \alpha_{2}}{\partial x_{2 e}} \frac{\partial \alpha_{1}}{\partial x_{1 e}}\right) w_{e}-\frac{\partial \alpha_{2}}{\partial x_{2 e}} \Delta_{e}
$$

which will be used in stability analysis of the closed loop system. It is noted that the virtual control $\alpha_{3}$ is a smooth function of $x_{1 e}, \xi_{1}, x_{2 e}, \xi_{2}, x_{3 e}$ since $x_{2}=x_{2 e}+\alpha_{1}$ and $x_{3}=x_{3 e}+\alpha_{2}$.

\subsection{Step 4}

This is the final step. The actual control input $i_{H}$ will be designed to regulate $x_{4 e}$ to a small neighborhood of the origin. To do so, differentiating both sides of (21) along the solutions of the last equation of the entire system (11) gives

$$
\begin{gathered}
\dot{x}_{4 e}=\frac{\partial g_{3}}{\partial x_{3}}\left(-\theta_{31} x_{2}-\theta_{32} x_{3}+\theta_{33} g_{3}\left(x_{3}, x_{4}\right)\right)+\frac{\partial g_{3}}{\partial x_{4}}\left(-\theta_{41} x_{4}+\theta_{42} i_{H}\right)-\frac{\partial \alpha_{3}}{\partial \xi_{1}} \dot{\xi}_{1}-\frac{\partial \alpha_{3}}{\partial \xi_{2}} \dot{\xi}_{2}- \\
\frac{\partial \alpha_{3}}{\partial x_{1 e}}\left(-k_{11} x_{1 e}+x_{2 e}+w_{e}\right)-\frac{\partial \alpha_{3}}{\partial x_{2 e}}\left(-x_{1 e}-k_{21} x_{2 e}+\theta_{21} x_{3 e}-\frac{\partial \alpha_{1}}{\partial x_{1 e}} w_{e}+\Delta_{e}\right)- \\
\frac{\partial \alpha_{3}}{\partial x_{3 e}}\left(-\theta_{21} x_{2 e}-k_{31} x_{3 e}+\theta_{33} x_{4 e}-\left(\frac{\partial \alpha_{2}}{\partial x_{1 e}}-\frac{\partial \alpha_{2}}{\partial x_{2 e}} \frac{\partial \alpha_{1}}{\partial x_{1 e}}\right) w_{e}-\frac{\partial \alpha_{2}}{\partial x_{2 e}} \Delta_{e}\right) .
\end{gathered}
$$


From (25), we choose the actual control $i_{H}$ as follows

$$
\begin{aligned}
i_{H}= & \frac{1}{\theta_{42} \frac{\partial g_{3}}{\partial x_{4}}}\left(-\theta_{33} x_{3 e}-k_{41} x_{4 e}+\theta_{41} x_{4}-\frac{\partial g_{3}}{\partial x_{3}}\left(-\theta_{31} x_{2}-\theta_{32} x_{3}+\theta_{33} g_{3}\left(x_{3}, x_{4}\right)\right)+\right. \\
& \frac{\partial \alpha_{3}}{\partial \xi_{1}} \dot{\xi}_{1}+\frac{\partial \alpha_{3}}{\partial \xi_{2}} \dot{\xi}_{2}+\frac{\partial \alpha_{3}}{\partial x_{1 e}}\left(-k_{11} x_{1 e}+x_{2 e}\right)+\frac{\partial \alpha_{3}}{\partial x_{2 e}}\left(-x_{1 e}-k_{21} x_{2 e}+\theta_{21} x_{3 e}\right)+ \\
& \left.\frac{\partial \alpha_{3}}{\partial x_{3 e}}\left(-\theta_{21} x_{2 e}-k_{31} x_{3 e}+\theta_{33} x_{4 e}\right)\right)
\end{aligned}
$$

where $k_{41}$ is a positive constant. It should be noted that since $P_{H S}-\left|P_{H}\right|=C_{H 3}\left(\bar{P}_{H s}-\left|x_{3}\right|\right) \geq \epsilon_{1}$, see Subsection 2.1 just below equation (3), and that

$$
\frac{\partial g_{3}}{\partial x_{4}}=\sqrt{\bar{P}_{H S}-x_{3} h\left(x_{4}, a, b\right)}-\frac{x_{3} x_{4} \frac{\partial h\left(x_{4}, a, b\right)}{\partial x_{4}}}{2 \sqrt{\bar{P}_{H S}-x_{3} h\left(x_{4} a, b\right)}} .
$$

From properties of the $p$-times differentiable signum function $h\left(x_{4}, a, b\right)$, we can see that there exists a strictly positive constant $\epsilon_{2}$ such that $\left|\frac{\partial g_{3}}{\partial x_{4}}\right| \geq \epsilon_{2}$. This means that the control $i_{H}$ given in (26) is well-defined. Substituting (26) into (25) results in the fourth closed loop sub-system

$$
\begin{gathered}
\dot{x}_{4 e}=-\theta_{33} x_{3 e}-k_{41} x_{4 e}-\left(\frac{\partial \alpha_{3}}{\partial x_{1 e}}-\frac{\partial \alpha_{3}}{\partial x_{2 e}} \frac{\partial \alpha_{1}}{\partial x_{1 e}}-\frac{\partial \alpha_{3}}{\partial x_{3 e}} \frac{\partial \alpha_{2}}{\partial x_{1 e}}+\frac{\partial \alpha_{3}}{\partial x_{3 e}} \frac{\partial \alpha_{2}}{\partial x_{2 e}} \frac{\partial \alpha_{1}}{\partial x_{1 e}}\right) w_{e}- \\
\left(\frac{\partial \alpha_{3}}{\partial x_{2 e}}-\frac{\partial \alpha_{3}}{\partial x_{3 e}} \frac{\partial \alpha_{3}}{\partial x_{2 e}}\right) \Delta_{e}
\end{gathered}
$$

The control design has been completed. Stability analysis of the closed loop system consisting of four closed loop sub-systems (16), (20), (24) and (28) is given in Appendix C. An appropriate choice of the control and observer gains $k_{11}, k_{12}, k_{21}, k_{22}, k_{31}$, and $k_{41}$ to avoid actuator saturations is given in the next section. For convenience of the reader, we summarize all the formulae that need to implement the proposed controller in Table 1. We now state the main result of the paper in the following theorem.

Theorem 1. Under the assumption that $z(t)$ and $\tilde{\Delta}\left(t, x_{H}, z, \dot{x}_{H}, \dot{z}\right)$ and their derivatives are bounded, the control input $i_{H}$ given in (26) and the disturbance observers given in (15) and (19) solve the control objective. In particular, the closed loop system consisting of (16) , (20), (24) and (28) is forward complete, and the active heave compensation error $x_{1 e}(t)=x_{1}(t)+z(t)-L$ exponentially converges to a small value. This value can be made arbitrarily small by choosing sufficiently large control and observer gains $k_{11}, k_{12}, k_{21}, k_{22}, k_{31}$, and $k_{41}$. If the derivatives of $z(t)$ and $\tilde{\Delta}\left(t, x_{H}, z, \dot{x}_{H}, \dot{z}\right)$ are zero, the active heave compensation error $x_{1 e}(t)=x_{1}(t)+z(t)-L$ exponentially converges to zero.

Proof. See Appendix C. 
Step 1:

$$
\begin{aligned}
& x_{1 e}=x_{1}+z(t)-L, \\
& x_{2 e}=x_{2}-\alpha_{1} \\
& \alpha_{1}=-k_{11} x_{1 e}-\hat{w} \\
& \hat{w}=\xi_{1}+k_{12} x_{1 e} \\
& \dot{\xi}_{1}=-k_{12} \xi_{1}-k_{12}\left(x_{2 e}+\alpha_{1}+k_{12} x_{1 e}\right) .
\end{aligned}
$$

Step 2:

Step 3:

$$
\begin{aligned}
& x_{3 e}=x_{3}-\alpha_{2}, \\
& \alpha_{2}=\frac{1}{\theta_{21}}\left(-x_{1 e}-k_{21} x_{2 e}+\theta_{22}\left(x_{2 e}+\alpha_{1}\right)+\frac{\partial \alpha_{1}}{\partial x_{1 e}}\left(-k_{11} x_{1 e}+x_{2 e}\right)+\frac{\partial \alpha_{1}}{\partial \xi_{1}} \dot{\xi}_{1}-\hat{\Delta}\right), \\
& \hat{\Delta}=\xi_{2}+k_{22} x_{2 e}, \\
& \dot{\xi}_{2}=-k_{22} \xi_{2}-k_{22}\left(\theta_{21}\left(x_{3 e}+\alpha_{2}\right)-\theta_{22}\left(x_{2 e}+\alpha_{1}\right)-\frac{\partial \alpha_{1}}{\partial x_{1 e}}\left(-k_{11} x_{1 e}+x_{2 e}\right)-\frac{\partial \alpha_{1}}{\partial \xi_{1}} \dot{\xi}_{1}\right) .
\end{aligned}
$$

Step 4:

$$
\begin{aligned}
x_{4 e}= & g_{3}\left(x_{3}, x_{4}\right)-\alpha_{3}, \\
\alpha_{3}= & \frac{1}{\theta_{33}}\left(-\theta_{21} x_{2 e}-k_{31} x_{3 e}+\theta_{31} x_{2}+\theta_{32} x_{3}+\frac{\partial \alpha_{2}}{\partial x_{1 e}}\left(-k_{11} x_{1 e}+x_{2 e}\right)+\frac{\partial \alpha_{2}}{\partial \xi_{1}} \dot{\xi}_{1}+\right. \\
& \left.\frac{\partial \alpha_{2}}{\partial x_{2 e}}\left(-x_{1 e}-k_{21} x_{2 e}+\theta_{21} x_{3 e}\right)+\frac{\partial \alpha_{2}}{\partial \xi_{2}} \dot{\xi}_{2}\right) .
\end{aligned}
$$

$$
\begin{aligned}
i_{H}= & \frac{1}{\theta_{42} \frac{\partial g_{3}}{\partial x_{4}}}\left(-\theta_{33} x_{3 e}-k_{41} x_{4 e}+\theta_{41} x_{4}-\frac{\partial g_{3}}{\partial x_{3}}\left(-\theta_{31} x_{2}-\theta_{32} x_{3}+\theta_{33} g_{3}\left(x_{3}, x_{4}\right)\right)+\right. \\
& \frac{\partial \alpha_{3}}{\partial \xi_{1}} \dot{\xi}_{1}+\frac{\partial \alpha_{3}}{\partial \xi_{2}} \dot{\xi}_{2}+\frac{\partial \alpha_{3}}{\partial x_{1 e}}\left(-k_{11} x_{1 e}+x_{2 e}\right)+\frac{\partial \alpha_{3}}{\partial x_{2 e}}\left(-x_{1 e}-k_{21} x_{2 e}+\theta_{21} x_{3 e}\right)+ \\
& \left.\frac{\partial \alpha_{3}}{\partial x_{3 e}}\left(-\theta_{21} x_{2 e}-k_{31} x_{3 e}+\theta_{33} x_{4 e}\right)\right) .
\end{aligned}
$$

Table 1. Summary of formulae for control implementation.

\section{Determination of control and observer gains}

The control and observer gains $k_{11}, k_{12}, k_{21}, k_{22}, k_{31}$, and $k_{41}$ directly affect performance of the closed loop system. The larger these gains are, the faster the response of the closed loop system is with the trade-off of a larger control effort at the transient response time, and vice-versa. Unfortunately, the current input $i_{H}$ cannot be arbitrarily large for any hydraulic systems used in an active compensation system in practice. This means that we need to choose the above control and observer gains such that they result in an as fast as possible response of the closed loop system, and avoidance of control saturation, i.e. the current input $i_{H}$ must lie in a certain range, say $\left|i_{H}\right| \leq i_{H}^{\max }$ where $i_{H}^{\max }$ is the maximum current inputting to the hydraulic system with an assumption of a symmetric current input hydraulic system. For a nonsymmetric current input hydraulic system, the following discussion can be carried out similarly. Moreover, each hydraulic 
system has a limited bandwidth. This means that if we force a hydraulic system to response too fast, the hydraulic system will not properly response to the commanded input, rather it just oscillates/vibrates.

In general, actuator saturation deteriorates the overall performance of the closed loop system, and can even destabilize the closed loop system. These problems are difficult to deal with but are important to take care of. In many cases, global stabilization and/or tracking result cannot be achieved when actuators are subject to saturation. For example, consider the scalar system

$$
\dot{x}=x^{2}+u
$$

with $x$ the state and $u$ the control. For this system, no bounded control $u$ can globally exponentially/asymptotically stabilize the system at the origin. In fact, any bounded control $u$ can result in a finite escape time. To illustrate the idea of dealing with control saturation, we consider a problem of stabilizing the scalar system (29). First, we design a control as

$$
u=-k x-x^{2}
$$

where the control gain $k$ is a positive constant. This control results in a closed loop system

$$
\dot{x}=-k x
$$

which is globally exponentially stable at the origin. Now assume that the control $u$ must lie in a certain range due to the actuator saturation, say $|u(t)| \leq u^{\max }, \forall t \geq t_{0} \geq 0$ where $u^{\max }$ is the maximum magnitude of the control input $u$. Given the initial value $x\left(t_{0}\right)$ and $u^{\max }$ with a feasible assumption that $u^{\max }$ is strictly larger than $x^{2}\left(t_{0}\right)$, we need to find the control gain $k$ such that $|u(t)| \leq u^{\max }, \forall t \geq t_{0} \geq 0$. From (31), we have $x(t)=x\left(t_{0}\right) e^{-k\left(t-t_{0}\right)}$, which implies that

$$
|x(t)| \leq\left|x\left(t_{0}\right)\right|, \forall t \geq t_{0} \geq 0 .
$$

Therefore, from (30) and (32), we have

$$
|u(t)| \leq k|x(t)|+x^{2}(t) \leq k\left|x\left(t_{0}\right)\right|+x^{2}\left(t_{0}\right), \forall t \geq t_{0} \geq 0 .
$$

Since we want $|u(t)| \leq u^{\max }, \forall t \geq t_{0} \geq 0$, from (34) it is sufficient to choose the control gain $k$ as

$$
k\left|x\left(t_{0}\right)\right|+x^{2}\left(t_{0}\right) \leq u^{\max } \Rightarrow k \leq \frac{u^{\max }-x^{2}\left(t_{0}\right)}{\left|x\left(t_{0}\right)\right|}, \forall t \geq t_{0} \geq 0 .
$$

Since the feasible condition (i.e. $u^{\max }$ is strictly larger than $\left.x^{2}\left(t_{0}\right)\right)$ implies that $u^{\max }-x^{2}\left(t_{0}\right)$ is strictly positive, we can always find a positive constant $k$ such that the condition (34) holds. In practice, we need to provide the maximum value of $\left|x\left(t_{0}\right)\right|$ satisfying the feasible condition to determine a range of the control gain $k$ must be lie in to stabilize the system (29) at the origin, and to avoid the control saturation. The condition (34) also implies that the control $u$ with the control gain $k$ satisfying (34) can only provide a local (not global) exponential stabilization solution. In literature, only restrictive tracking control and stabilization can be achieved for many systems including one consisting of a simple integrator chain (Teel, 1992).

We now discuss how to use the proposed controller given in Section 3 when both actuator magnitude and rate saturations present in the active heave compensation in question. We first deal with the case where the actuator magnitude saturation presents then move to the case where the actuator rate saturation is imposed. 


\subsection{Actuator magnitude saturation case}

Motivated by the aforementioned example, we will first set $\left|i_{H}\right| \leq i_{H}^{\max }$, then find restrictions on the control and observer gains, and the initial conditions such that the condition $\left|i_{H}\right| \leq i_{H}^{\max }$ holds. From (26) , the condition $\left|i_{H}\right| \leq i_{H}^{\max }$ is equivalent to

$$
\begin{aligned}
& \mid \frac{1}{\theta_{42} \frac{\partial g_{3}}{\partial x_{4}}}\left(-\theta_{33} x_{3 e}-k_{41} x_{4 e}+\theta_{41} x_{4}-\frac{\partial g_{3}}{\partial x_{3}}\left(-\theta_{31} x_{2}-\theta_{32} x_{3}+\theta_{33} g_{3}\left(x_{3}, x_{4}\right)\right)+\right. \\
& \frac{\partial \alpha_{3}}{\partial \xi_{1}} \dot{\xi}_{1}+\frac{\partial \alpha_{3}}{\partial \xi_{2}} \dot{\xi}_{2}+\frac{\partial \alpha_{3}}{\partial x_{1 e}}\left(-k_{11} x_{1 e}+x_{2 e}\right)+\frac{\partial \alpha_{3}}{\partial x_{2 e}}\left(-x_{1 e}-k_{21} x_{2 e}+\theta_{21} x_{3 e}\right)+ \\
& \left.\frac{\partial \alpha_{3}}{\partial x_{3 e}}\left(-\theta_{21} x_{2 e}-k_{31} x_{3 e}+\theta_{33} x_{4 e}\right)\right) \mid \leq i_{H}^{\max }
\end{aligned}
$$

To find a range that the control and observer gains should lie in, with a note that $\left|\frac{\partial g_{3}}{\partial x_{4}}\right| \geq \epsilon_{2}$, see Subsection 3.4, we need to calculate upper bounds of all the terms in the left-hand side of (35). These bounds depend on the control and observer gains, and the initial conditions. After the bounds are substituted into the left-hand side of (35), we will be able to find a feasible range of the control and observer gains. We first find the upper bounds of $x_{1 e}, x_{2 e}, x_{3 e}, x_{4 e}$, $w_{e}$ and $\Delta_{e}$. Based on these values, we are then be able to find upper bounds of all the terms in the left-hand side of (35). As such, integrating both sides of (56), see Appendix $\mathrm{C}$, we have $V(t) \leq\left(V\left(t_{0}\right)-\frac{\lambda}{c}\right) e^{-c\left(t-t_{0}\right)}+\frac{\lambda}{c}, \forall t \geq t_{0} \geq 0$, which implies that $V(t) \leq V\left(t_{0}\right)+\frac{\lambda}{c}$. From the definition of $V(t)$, see (51) in Appendix C, we have

$$
\begin{aligned}
&\left(x_{1 e}^{2}(t)+\right.\left.x_{2 e}^{2}(t)+x_{3 e}^{2}(t)+x_{4 e}^{2}(t)+\delta_{1} w_{e}^{2}(t)+\delta_{2} \Delta_{e}^{2}(t)\right) \leq \\
&\left(x_{1 e}^{2}\left(t_{0}\right)+x_{2 e}^{2}\left(t_{0}\right)+x_{3 e}^{2}\left(t_{0}\right)+x_{4 e}^{2}\left(t_{0}\right)+\delta_{1} w_{e}^{2}\left(t_{0}\right)+\delta_{2} \Delta_{e}^{2}\left(t_{0}\right)\right)+\frac{\lambda}{c}:=\Omega_{0} .
\end{aligned}
$$

From (36) and the definition of $x_{1 e}, x_{2 e}, x_{3 e}, x_{4 e}, w_{e}$ and $\Delta_{e}$, we first calculate the following immediate upper bounds of $\alpha_{1}, x_{2}, \xi_{1}, \dot{\xi}_{1}, \alpha_{2}, x_{3}, \xi_{2}, \dot{\xi}_{2}, \alpha_{3}$ and $x_{4}$ as follows:

$$
\begin{aligned}
& \left|x_{1 e}\right| \leq \Omega_{0},\left|x_{2 e}\right| \leq \Omega_{0},\left|x_{3 e}\right| \leq \Omega_{0},\left|x_{4 e}\right| \leq \Omega_{0},\left|w_{e}\right| \leq \frac{\Omega_{0}}{\delta_{1}},\left|\Delta_{e}\right| \leq \frac{\Omega_{0}}{\delta_{2}} \\
& \left|\alpha_{1}\right| \leq k_{11}\left|x_{1 e}\right|+|\hat{w}| \leq k_{11}\left|x_{1 e}\right|+\left|w_{e}\right|+|w| \leq k_{11} \Omega_{0}+\frac{\Omega_{0}}{\delta_{1}}+C_{w}:=\alpha_{1}^{M}, \\
& \left|x_{2}\right| \leq\left|x_{2 e}\right|+\left|\alpha_{1}\right| \leq \Omega_{0}+k_{11} \Omega_{0}+\frac{\Omega_{0}}{\delta_{1}}+C_{w}:=x_{2}^{M}, \\
& \left|\xi_{1}\right| \leq|\hat{w}|+k_{12}\left|x_{1 e}\right| \leq \frac{\Omega_{0}}{\delta_{1}}+C_{w}+k_{12} \Omega_{0}:=\xi_{1}^{M}, \\
& \left|\dot{\xi}_{1}\right| \leq k_{12}\left|\xi_{1}\right|+k_{12}\left(\left|x_{2 e}\right|+\left|\alpha_{1}\right|+k_{12}\left|x_{1 e}\right|\right):=\dot{\xi}_{1}^{M} \\
& |\hat{\Delta}| \leq|\Delta|+\left|\Delta_{e}\right| \leq C_{\Delta}+\frac{\Omega_{0}}{\delta_{2}}:=\hat{\Delta}^{M}, \\
& \left|\alpha_{2}\right| \leq \frac{1}{\left|\theta_{21}\right|}\left(\left|x_{1 e}\right|+k_{21}\left|x_{2 e}\right|+\left|\theta_{22}\right|\left(\left|x_{2 e}\right|+\left|\alpha_{1}\right|\right)+\left|\frac{\partial \alpha_{1}}{\partial x_{1 e}}\right|\left(k_{11}\left|x_{1 e}\right|+\left|x_{2 e}\right|\right)+\right. \\
& \left.\left|\frac{\partial \alpha_{1}}{\partial \xi_{1}}\right|\left|\dot{\xi}_{1}\right|+|\hat{\Delta}|\right):=\alpha_{2}^{M} \\
& \left|x_{3}\right| \leq\left|x_{3 e}\right|+\left|\alpha_{2}\right|:=x_{3}^{M} \\
& \left|\xi_{2}\right| \leq|\hat{\Delta}|+k_{22}\left|x_{2 e}\right|:=\xi_{2}^{M}
\end{aligned}
$$




$$
\begin{aligned}
\left|\dot{\xi}_{2}\right| & \leq k_{22}\left|\xi_{2}\right|+k_{22}\left(\left|\theta_{21}\right|\left(\left|x_{3 e}\right|+\left|\alpha_{2}\right|\right)+\left|\theta_{22}\right|\left(\left|x_{2 e}\right|+\left|\alpha_{1}\right|\right)+\right. \\
\mid & \left.\left|\frac{\partial \alpha_{1}}{\partial x_{1 e}}\right|\left(k_{11}\left|x_{1 e}\right|+\left|x_{2 e}\right|\right)+\left|\frac{\partial \alpha_{1}}{\partial \xi_{1}}\right|\left|\dot{\xi}_{1}\right|\right):=\dot{\xi}_{2}^{M} \\
\left|\alpha_{3}\right| & \leq \frac{1}{\left|\theta_{33}\right|}\left(\left|\theta_{21}\right|\left|x_{2 e}\right|+k_{31}\left|x_{3 e}\right|+\left|\theta_{31}\right|\left|x_{2}\right|+\left|\theta_{32}\right|\left|x_{3}\right|+\left|\frac{\partial \alpha_{2}}{\partial x_{1 e}}\right|\left(k_{11}\left|x_{1 e}\right|+\left|x_{2 e}\right|\right)+\right. \\
& \left.\left|\frac{\partial \alpha_{2}}{\partial \xi_{1}}\right|\left|\dot{\xi}_{1}\right|+\left|\frac{\partial \alpha_{2}}{\partial x_{2 e}}\right|\left(\left|x_{1 e}\right|+k_{21}\left|x_{2 e}\right|+\left|\theta_{21}\right|\left|x_{3 e}\right|\right)+\left|\frac{\partial \alpha_{2}}{\partial \xi_{2}}\right|\left|\dot{\xi}_{2}\right|\right):=\alpha_{3}^{M}, \\
\left|x_{4}\right| & \leq\left|x_{4 e}\right|+\left|g_{3}\left(x_{3}, x_{4}\right)\right|:=x_{4}^{M}
\end{aligned}
$$

where $C_{w}$ and $\Delta^{M}$ is the maximum value of $|w|$ and $|\Delta|$, respectively. It is noted that all the terms involving the partial derivatives can be easily upper bounded based on (37). Now the condition (35) can be written as

$$
\begin{aligned}
& \frac{1}{\left|\theta_{42}\right|\left|\frac{\partial g_{3}}{\partial x_{4}}\right|}\left(\left|\theta_{33}\right|\left|x_{3 e}\right|+k_{41}\left|x_{4 e}\right|+\left|\theta_{41}\right|\left|x_{4}\right|+\left|\frac{\partial g_{3}}{\partial x_{3}}\right|\left(\left|\theta_{31}\right|\left|x_{2}\right|+\left|\theta_{32}\right|\left|x_{3}\right|+\left|\theta_{33}\right|\left|g_{3}\left(x_{3}, x_{4}\right)\right|\right)+\right. \\
& \left|\frac{\partial \alpha_{3}}{\partial \xi_{1}}\right|\left|\dot{\xi}_{1}\right|+\left|\frac{\partial \alpha_{3}}{\partial \xi_{2}} \dot{\xi}_{2}+\frac{\partial \alpha_{3}}{\partial x_{1 e}}\right|\left(k_{11}\left|x_{1 e}\right|+\left|x_{2 e}\right|\right)+\left|\frac{\partial \alpha_{3}}{\partial x_{2 e}}\right|\left(\left|x_{1 e}\right|+k_{21}\left|x_{2 e}\right|+\left|\theta_{21}\right|\left|x_{3 e}\right|\right)+ \\
& \left.\left|\frac{\partial \alpha_{3}}{\partial x_{3 e}}\right|\left(\left|\theta_{21}\right|\left|x_{2 e}\right|+k_{31}\left|x_{3 e}\right|+\left|\theta_{33}\right|\left|x_{4 e}\right|\right)\right) \leq i_{H}^{\max }
\end{aligned}
$$

Substituting (37) into (38) results in an inequality that all the control and observer gains $k_{11}$, $k_{12}, k_{21}, k_{22}, k_{31}$, and $k_{41}$ must be satisfied to guarantee that no saturation of the current $i_{H}$ occurs. Since an explicit expression of the range that all the control and observer gains should lie in is pretty complicated, we do not write it down here. However, a simple computer program can be written to give the explicit range of the control and observer gains since it mainly involves with a recursive substitution.

\subsection{Actuator rate saturation case}

For this case, we make a practical observation that in an hydraulic system the current input $i_{H}$ can be changed arbitrarily fast (Yao et al., 2001) and that the rate limitation is placed on the velocity, $x_{2}$, of the main piston. As such, we just need to impose a constraint $\left|x_{2}(t)\right| \leq R_{x_{2}}^{M}$ where $R_{x_{2}}^{M}$ is the maximum allowable absolute value of the main piston velocity, $x_{2}$. Using (37), we can deduce a condition on the control and observer gains to ensure that the rate saturation does not occur as follows:

$$
\left|x_{2}\right| \leq\left|x_{2 e}\right|+\left|\alpha_{1}\right| \leq \Omega_{0}+k_{11} \Omega_{0}+\frac{\Omega_{0}}{\delta_{1}}+C_{w}:=x_{2}^{M} \leq R_{x_{2}}^{M}
$$

which is an additional condition to those conditions derived in Subsection (4.1) to choose the control and observer gains $k_{11}, k_{12}, k_{21}, k_{22}, k_{31}$, and $k_{41}$ so that the actuator rate and magnitude saturations are avoided.

\section{Simulations}

To illustrate the effectiveness of the controller proposed in the previous section, we carry out some simulations in this section. The parameters of the hydraulic system are taken based on (Yao et 
al., 2001) as follows: $m_{H}=1000 \mathrm{~kg}, A_{H}=0.65 \mathrm{~m}^{2}, b_{H}=40 \mathrm{~N} /(\mathrm{m} / \mathrm{s}), 4 \beta_{H e} / V_{H}=4.53 \times 10^{8} \mathrm{~N} / \mathrm{m}^{5}$, $C_{H D}=2.21 \times 10^{-14} \mathrm{~m}^{5} / \mathrm{Ns}, C_{H D} w_{H} / \sqrt{\rho}=3.42 \times 10^{-5} \mathrm{~m}^{3} \sqrt{N} s, P_{H S}=10342500 P a, k_{H v}=$ $0.0324, \tau_{H v}=0.00636$. The scale factors are taken as $C_{H 3}=6 \times 10^{5}, C_{H 4}=5 \times 10^{-7}$ to scale $P_{H S}$ down and $\tau_{H v}$ up as discussed in Subsection 2.1. The riser parameters are taken from (Korde, 1998) as follows: length $L=3832 \mathrm{~m}$, diameter $d_{r}=0.14 \mathrm{~m}$, density $\rho_{r}=8200 \mathrm{~kg} / \mathrm{m}^{3}$, Young's modulus $E_{r}=2 \times 10^{8} \mathrm{~kg} / \mathrm{m}^{2}$, initial tension $T_{o r}=100 \mathrm{KN}$. Therefore, the force (disturbance), $\tilde{\Delta}$ acting from the riser to the piston of the hydraulic system is (Korde, 1998):

$$
\tilde{\Delta}=T_{o r}+E_{r} A_{r}\left(\sum_{n=1}^{N_{m}} \mp n \pi C_{n}+\delta\right)
$$

with $C_{n}$ being generated by

$$
\ddot{C}_{n}+c_{d} \dot{C}_{n}+\frac{E_{r} A_{r} n^{2} \pi^{2}}{\rho_{r} L^{2}} C_{n}=\mp \frac{2}{n \pi}\left(c_{d} \dot{\delta}+\ddot{\delta}\right)
$$

where $\delta=z-x_{H}-L, A_{r}=\frac{\pi d_{r}^{2}}{4}$, the damping coefficient $c_{d}=0.01 \mathrm{~m}^{3} / \mathrm{s}$, and the notation $\mp$ takes the positive sign if $n=2,4,6, \ldots$ and the negative sign if $n=1,3,5, \ldots$, and $N_{m}$ is number of modes. The vertical (heave) motion of the vessel $z(t)$ can be represented as a sum of sinusoids at different frequencies, amplitudes and phases as follows, see (Fossen, 1994) and (Lloyd, 1998):

$$
z(t)=L+\sum_{i=1}^{N_{w}}\left(A_{w i} k_{w i} \sin \left(w_{w i} t-\varphi_{w i}\right)\right)
$$

where $L$ is included since $z(t)$ is coordinated in the earth-fixed frame, the amplitude $A_{w i}$, coefficient $k_{w i}$, frequency $w_{w i}$, phase $\varphi_{w i}$ of the wave $i^{t h}$ are given by

$$
\begin{aligned}
& w_{w i}=w_{m}+\frac{w_{m}-w_{M}}{N_{w}} i, \quad S_{w i}=\frac{1.25}{4} \frac{w_{o}^{4}}{w_{w i}^{5}} H_{s w}^{2} e^{-1.25\left(\frac{w_{o}}{w_{w i}}\right)^{4}} \\
& A_{w i}=\sqrt{2 S_{w i} \frac{w_{m i}-w_{M i}}{N_{w}}}, \quad k_{w i}=\frac{w_{w i}^{2}}{9.8}, \quad \varphi_{w i}=2 \pi \operatorname{rand}() .
\end{aligned}
$$

In (43), the minimum and maximum wave frequencies are $w_{m}=0.2 \mathrm{rand} / \mathrm{s}, w_{M}=2.5 \mathrm{rand} / \mathrm{s}$; the two-parameter Bretschneider spectrum $S_{w i}$ is used with the significant wave height $H_{s w}=4 \mathrm{~m}$; the modal frequency is $w_{o}=\frac{2 \pi}{T_{w}}$ with the period $T_{w}=7.8 ; N_{w}=10$; and $\operatorname{rand}()$ is a random number between 0 and 1 .

The control and disturbance observer gains are chosen as $k_{11}=2, k_{12}=15, k_{21}=4, k_{22}=$ $25, k_{31}=8, k_{41}=12$. In the simulations, we take the number of modes in (40) as $N_{m}=5$, and initial values of $C_{n}$ in (41) are zero. To illustrate the effectiveness of the disturbance observers, we simulate the proposed controller in two cases. In the first case, the disturbance observers are switched on. The simulation result is presented in Figure 5 .

In Figure 5, the top sub-figure plots the error $x_{1 e}$, the bottom sub-figure plots the disturbance $\Delta$ and its estimate $\hat{\Delta}$. From the bottom sub-figure, we can see that the disturbance observer estimates the disturbance $\Delta$ quite well, see also Figure 6 for a short time presentation of $\Delta$ and $\hat{\Delta}$. This results in pretty small error $x_{1 e}$. It is noted that the error $x_{1 e}$ does not converge to zero as said Theorem 1 because $\Delta$ and $\ddot{z}(t)$ are time-varying.

In the second case, the disturbance observers are switched-off. The simulation result is presented in Figure 7. Explanation of the sub-figures is the same as that in the first case. From 

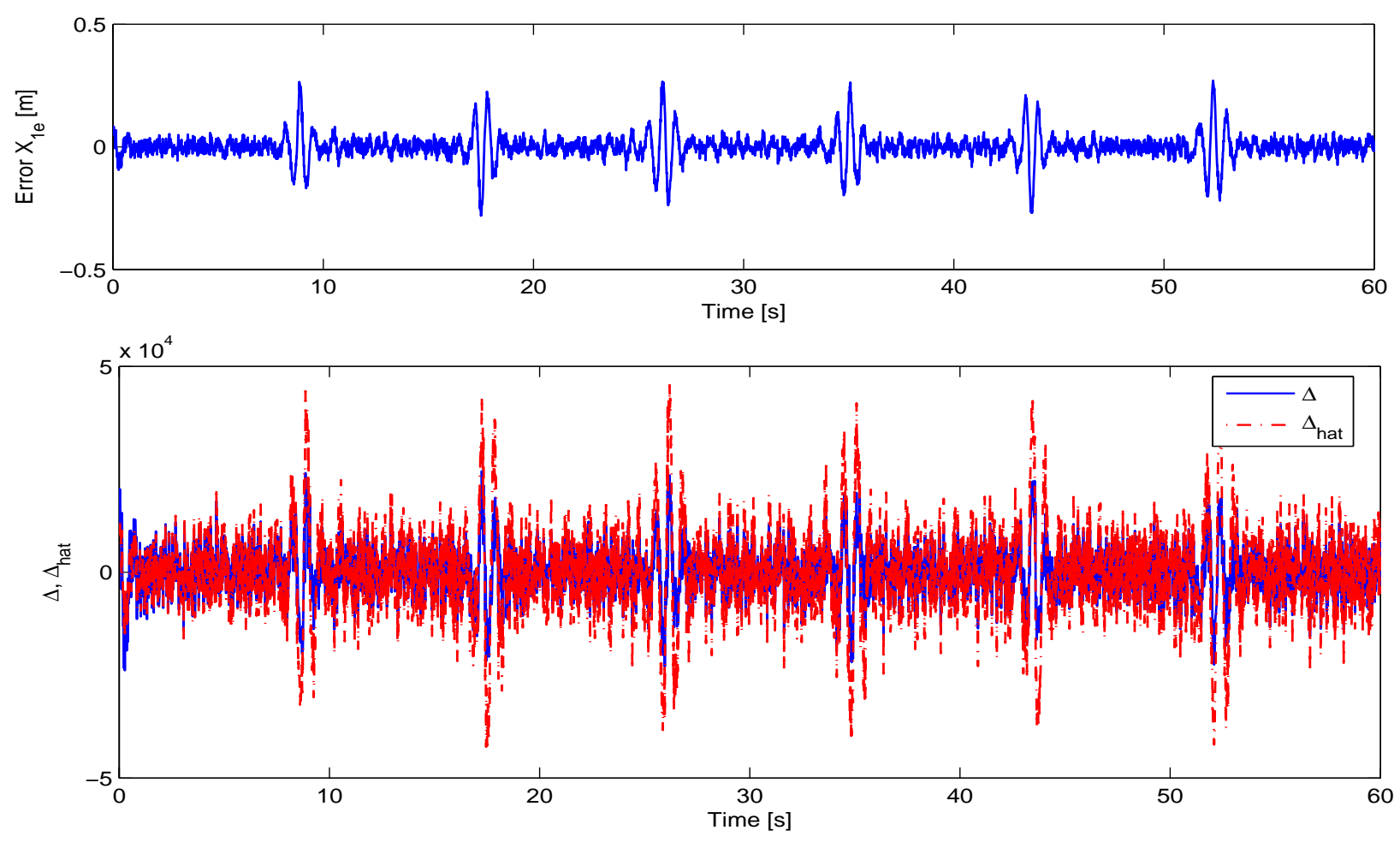

Figure 5: Simulation result with disturbance observers.

these sub-figures, we can see that when the disturbance observers are switched-off, the error $x_{1 e}$ is pretty large. This is because the controller does not compensate for the disturbances via the disturbance observers. The above simulation results support the fact in the control design that the disturbance observers play a vital role in the proposed controller for the active heave compensation system (i.e. to achieve a small $\left.\left|x_{1 e}\right|\right)$ considered in this paper.

\section{Conclusions}

A nonlinear controller has been designed for an active heave compensation system. One of the keys to success in the proposed method is the use of the disturbance observers, which are then properly embedded in the control design procedure. The heave velocity and the force acting between the riser/drill-string and the active heave compensation unit were well estimated by the disturbances observers. Future work includes extending the proposed control design technique in this paper to compensate for the local/small motion of the vessel/rig in $(x, y)$ plane as well to achieve fine tuning of riser/drill-string angles at the bottom and top ends since it is costly to use a dynamic positioning system to adjust small motion of the vessel/rig.

Acknowledgements: We thank the reviewers for their helpful comments, which help us improve the paper significantly. This work is supported by the ARC Discovery grant No. DP0774645.

Appendix A: Proof of Lemma 1. To prove Lemma 1, we calculate the derivative of 


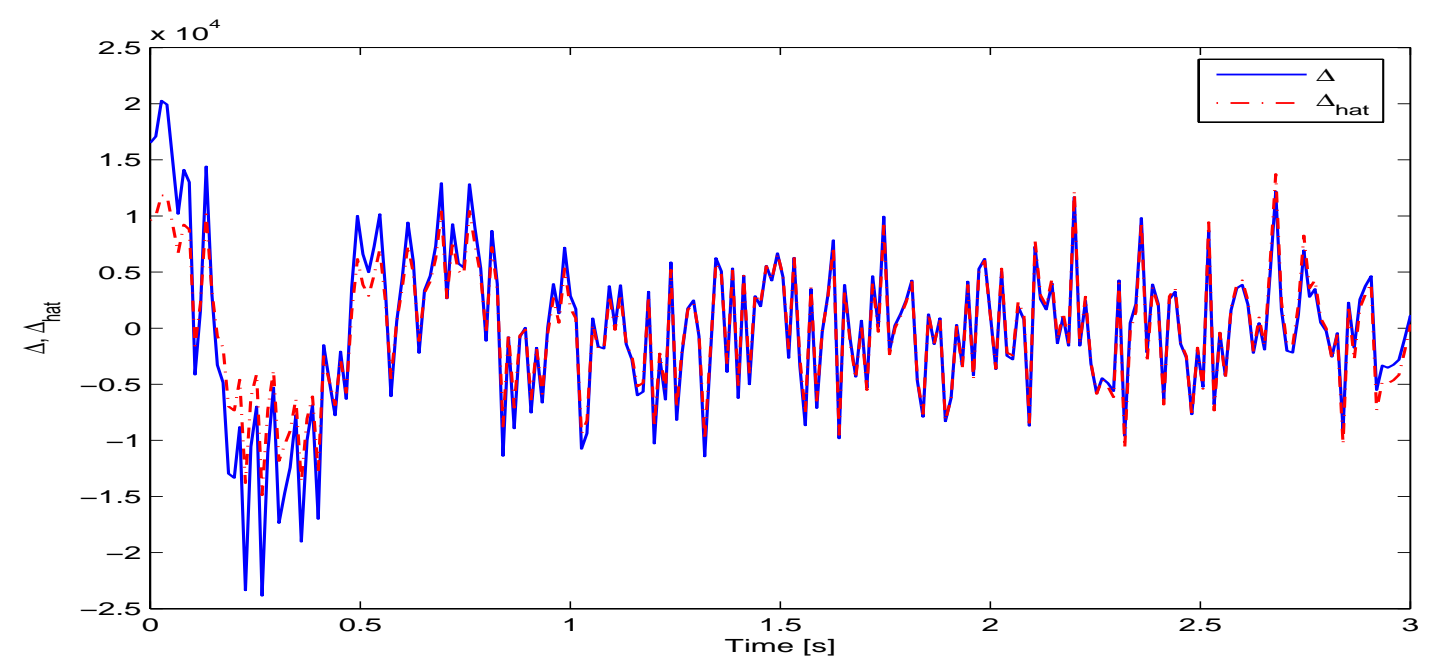

Figure 6: A short time presentation of $\Delta$ and $\hat{\Delta}$

$d_{e}(t, x)$ as follows

$$
\begin{aligned}
\dot{d}_{e}(t, x) & =\dot{d}(t, x)-(\dot{\xi}+\dot{\rho}(x)) \\
& =K(x)(\hat{d}(t, x)-\rho(x))+K(x)(f(x)+u+\rho(x))-K(x)(f(x)+u+d(t, x))+\dot{d}(t, x) \\
& =-K(x)(d(t, x)-\hat{d}(t, x))+\dot{d}(t, x) \\
& =-K(x) d_{e}(t, x)+\dot{d}(t, x) .
\end{aligned}
$$

Consider the Lyapunov function

$$
V_{e}=\frac{1}{2} d_{e}^{T}(t, x) d_{e}(t, x)
$$

whose first time derivative along the solutions of (44) satisfies

$$
\dot{V}_{e}=-\frac{1}{2} d_{e}^{T}(t, x)\left(K(x)+K^{T}(x)\right) d_{e}(t, x)+d_{e}^{T}(t, x) \dot{d}(t, x) .
$$

Since $K(x)$ is a positive definite matrix, there exists a positive constant $\varpi$ such that

$$
d_{e}^{T}(t, x)\left(K(x)+K^{T}(x)\right) d_{e}(t, x) \geq \varpi d_{e}^{T}(t, x) d_{e}(t, x), \forall x \in \mathbb{R} .
$$

Substituting (47) into (46) results in

$$
\begin{aligned}
\dot{V}_{e} & \leq-(\varpi-2 \varepsilon) V_{e}+\frac{1}{4 \varepsilon}\|\dot{d}(t, x)\|^{2} \\
& \leq-(\varpi-2 \varepsilon) V_{e}+\frac{1}{4 \varepsilon} C_{d}^{2}
\end{aligned}
$$

where we have used (45) and $\left|d_{e}^{T}(t, x) \dot{d}(t, x)\right| \leq \varepsilon\left\|d_{e}(t, x)\right\|+\frac{1}{4 \varepsilon}\|\dot{d}(t, x)\|$ with $\varepsilon$ a positive constant such that $\varpi-2 \varepsilon$ is strictly positive. From the second inequality of (48), it is seen that $V_{e}$ exponentially converges to a ball centered the origin with the radius $R_{V e}=\frac{C_{d}^{2}}{4 \varepsilon(\varpi-2 \varepsilon)}$ as long as the solutions $x(t)$ exist. The existence of the solutions $x(t)$ is to be guaranteed by the design 

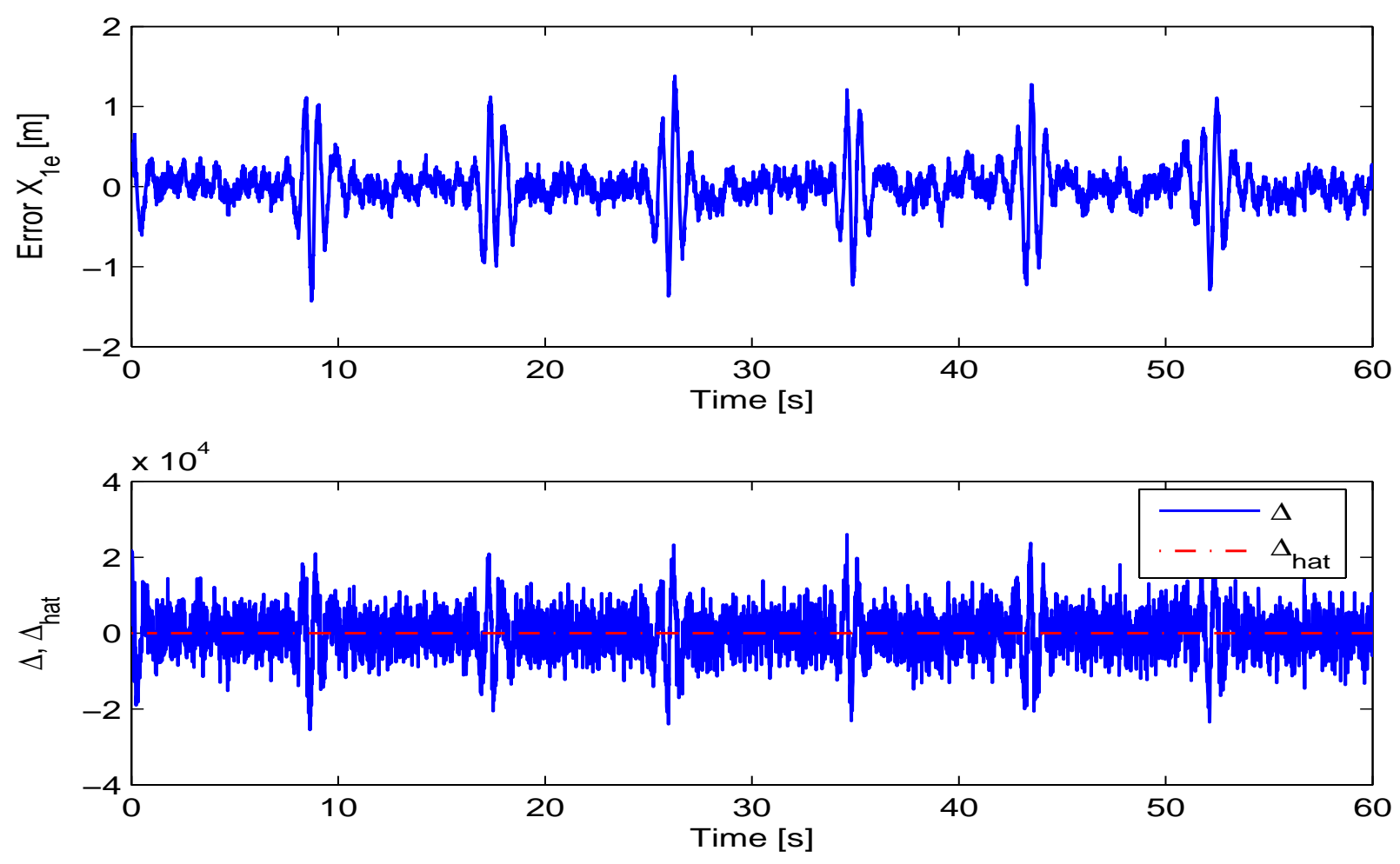

Figure 7: Simulation result without disturbance observers.

of the control input $u$. This in turn means that the disturbance error $d_{e}(t, x)$ exponentially converges to a ball centered at the origin with the radius $R_{d e}=\sqrt{\frac{1}{2 \varepsilon(\varpi-2 \varepsilon)}} C_{d}$. Since $\varpi$ can be chosen arbitrarily large by choosing the function $\rho(x)$, the radius $R_{d e}$ can be made arbitrarily small. In the case $C_{d}=0$, the radius $R_{V e}=R_{d e}=0$ meaning that the disturbance error $d_{e}(t, x)$ exponentially converges to zero.

Appendix B: Proof of Lemma 2. For the case $f(y)=y^{p}$, see (Do, 2007) for proof of Properties 1)-4). We here consider the case where $f(y)=y^{p}$ in (10) is replaced by $f(y)=e^{-1 / y}$. Properties 1)-3) can be proven in the same lines as in (Do, 2007). We focus on proof of Property $\left.4^{\prime}\right)$. We first note that $f^{(k)}(y)=\frac{\partial^{k} f(y)}{\partial y^{k}}=Q_{k}\left(\frac{1}{y}\right) e^{-\frac{1}{y}}$ where $Q_{k}\left(\frac{1}{y}\right)$ is a polynomial function of $\frac{1}{y}$, and $k$ is any positive integer. We will prove Property 4') by induction. First of all, we check that $f^{(1)}(0)=0$. It is clear that $\lim _{y \rightarrow 0^{-}} \frac{f(y)-f(0)}{y-0}=\lim _{y \rightarrow 0^{-}} \frac{0}{y}=0$. On the other hand, $\lim _{y \rightarrow 0^{+}} \frac{f(y)-f(0)}{y-0}=\lim _{y \rightarrow 0^{+}} \frac{e^{-\frac{1}{y}}}{y}=\lim _{\xi \rightarrow \infty} \frac{1}{e^{\xi}}=0$ where $\xi=\frac{1}{y}$ and we have used l'Hopital's rule. Since both left- and right-hand limits are equal to 0 , we have $f^{(1)}(0)=0$. Now assume that $f^{(k)}(0)=0$. We now compute $f^{(k+1)}(0)$. The left-hand limit is equal to 0 as above. The right hand limit is $\lim _{y \rightarrow 0^{+}} \frac{f^{(k)}(y)-f^{(k)}(0)}{y-0}=\lim _{y \rightarrow 0^{+}} \frac{f^{(k)}(y)-0}{y-0}=\lim _{y \rightarrow 0^{+}} \frac{1}{y} Q_{k}\left(\frac{1}{y}\right) e^{-\frac{1}{y}}=$ $\lim _{y \rightarrow 0^{+}} \tilde{Q}_{k}\left(\frac{1}{y}\right) e^{-\frac{1}{y}}=\lim _{\xi \rightarrow \infty} \frac{\tilde{Q_{k}(\xi)}}{e^{\xi}}=0$ (by l'Hopital's rule), where $\xi=\frac{1}{y}$ and $\tilde{Q}_{k}(\xi)=\xi Q_{k}(\xi)$ is another polynomial of $\frac{1}{y}$. Since both left- and right-hand limits are equal to 0 , we have $f^{(k+1)}(0)=0$, which completes proof of Property $\left.4^{\prime}\right)$.

Appendix C Proof of Theorem 1. For convenience, we rewrite the closed loop system 
consisting of (16), (20), (24) and (28) as follows:

$$
\begin{aligned}
& \dot{x}_{1 e}=-k_{11} x_{1 e}+x_{2 e}+w_{e} \\
& \dot{x}_{2 e}=-x_{1 e}-k_{21} x_{2 e}+\theta_{21} x_{3 e}-\phi_{1} w_{e}+\Delta_{e} \\
& \dot{x}_{3 e}=-\theta_{21} x_{2 e}-k_{31} x_{3 e}+\theta_{33} x_{4 e}-\phi_{2} w_{e}-\phi_{3} \Delta_{e} \\
& \dot{x}_{4 e}=-\theta_{33} x_{3 e}-k_{41} x_{4 e}-\phi_{4} w_{e}-\phi_{5} \Delta_{e} \\
& \dot{w}_{e}=-k_{12} w_{e}+\dot{w} \\
& \dot{\Delta}_{e}=-k_{22} \Delta_{e}+k_{22} \phi_{1} w_{e}+\dot{\Delta}
\end{aligned}
$$

where

$$
\begin{aligned}
\phi_{1} & =\frac{\partial \alpha_{1}}{\partial x_{1 e}}, \quad \phi_{2}=\left(\frac{\partial \alpha_{2}}{\partial x_{1 e}}-\frac{\partial \alpha_{2}}{\partial x_{2 e}} \frac{\partial \alpha_{1}}{\partial x_{1 e}}\right), \quad \phi_{3}=\frac{\partial \alpha_{2}}{\partial x_{2 e}} \\
\phi_{4} & =\left(\frac{\partial \alpha_{3}}{\partial x_{1 e}}-\frac{\partial \alpha_{3}}{\partial x_{2 e}} \frac{\partial \alpha_{1}}{\partial x_{1 e}}-\frac{\partial \alpha_{3}}{\partial x_{3 e}} \frac{\partial \alpha_{2}}{\partial x_{1 e}}+\frac{\partial \alpha_{3}}{\partial x_{3 e}} \frac{\partial \alpha_{2}}{\partial x_{2 e}} \frac{\partial \alpha_{1}}{\partial x_{1 e}}\right) \\
\phi_{5} & =\left(\frac{\partial \alpha_{3}}{\partial x_{2 e}}-\frac{\partial \alpha_{3}}{\partial x_{3 e}} \frac{\partial \alpha_{3}}{\partial x_{2 e}}\right) .
\end{aligned}
$$

To investigate stability of the closed loop system (49), we consider the following Lyapunov function candidate

$$
V=\frac{1}{2}\left(x_{1 e}^{2}+x_{2 e}^{2}+x_{3 e}^{2}+x_{4 e}^{2}+\delta_{1} w_{e}^{2}+\delta_{2} \Delta_{e}^{2}\right)
$$

where $\delta_{1}$ and $\delta_{2}$ are positive constants to be picked later. Differentiating both sides of (51) along the solutions of (49) gives

$$
\begin{aligned}
\dot{V} & =-k_{11} x_{1 e}^{2}-k_{21} x_{2 e}^{2}-k_{31} x_{3 e}^{2}-k_{41} x_{4 e}^{2}-\delta_{1} k_{12} w_{e}^{2}-\delta_{2} k_{22} \Delta_{e}^{2}+w_{e} x_{1 e}-\phi_{1} w_{e} x_{2 e}+\Delta_{e} x_{2 e} \\
& -\phi_{2} w_{e} x_{3 e}-\phi_{3} \Delta_{e} x_{3 e}-\phi_{4} w_{e} x_{4 e}-\phi_{5} \Delta_{e} x_{4 e}+\delta_{1} \dot{w} w_{e}+\delta_{2} k_{22} \phi_{1} w_{e} \Delta_{e}+\delta_{2} \dot{\Delta} \Delta_{e} .
\end{aligned}
$$

From the expressions of $\alpha_{1}, \alpha_{2}$ and $\alpha_{3}$, see (15), (19) and (23), there exist nonnegative constants $A_{i}, i=1, \ldots, 5$ such that

$$
\left|\phi_{i}\right| \leq A_{i}, i=1, \ldots, 5 \text {. }
$$

Using (53) and completion of squares, we can write (152) as

$$
\begin{aligned}
\dot{V} & \leq-\frac{k_{11}}{2} x_{1 e}^{2}-\frac{k_{21}}{2} x_{2 e}^{2}-\frac{k_{31}}{2} x_{3 e}^{2}-\frac{k_{41}}{2} x_{4 e}^{2} \\
& -\left(\delta_{1} k_{12}-\frac{1}{2 k_{11}}-\frac{A_{1}^{2}}{k_{21}}-\frac{A_{2}^{2}}{k_{31}}-\frac{A_{4}^{2}}{k_{41}}-\delta_{1} \epsilon_{1}-\frac{k_{22} A_{1} \delta_{2}}{4 \epsilon_{2}}\right) w_{e}^{2} \\
& -\left(\delta_{2} k_{22}-\frac{1}{k_{21}}-\frac{A_{3}^{2}}{k_{31}}-\frac{A_{5}^{2}}{k_{41}}-\epsilon_{2} k_{22} A_{1} \delta_{2}\right) \Delta_{e}^{2}+\frac{\delta_{1}}{4 \epsilon_{1}} \dot{w}^{2}+\frac{\delta_{2}}{4 \epsilon_{3}} \dot{\Delta}^{2}
\end{aligned}
$$

where $\epsilon_{i}, i=1,2,3$ are positive constants to be picked. Now for chosen control gains $k_{11}, k_{21}$, $k_{31}, k_{41}, k_{12}$ and $k_{22}$, we can always pick the positive constants $\delta_{1}, \delta_{2}$ and $\epsilon_{i}, i=1,2,3$ such that

$$
\begin{aligned}
& \left(\delta_{1} k_{12}-\frac{1}{2 k_{11}}-\frac{A_{1}^{2}}{k_{21}}-\frac{A_{2}^{2}}{k_{31}}-\frac{A_{4}^{2}}{k_{41}}-\delta_{1} \epsilon_{1}-\frac{k_{22} A_{1} \delta_{2}}{4 \epsilon_{2}}\right) \geq \frac{\gamma_{1}}{2}, \\
& \left(\delta_{2} k_{22}-\frac{1}{k_{21}}-\frac{A_{3}^{2}}{k_{31}}-\frac{A_{5}^{2}}{k_{41}}-\epsilon_{2} k_{22} A_{1} \delta_{2}\right) \geq \frac{\gamma_{2}}{2}
\end{aligned}
$$


where $\gamma_{1}$ and $\gamma_{2}$ are positive constants. Substituting (55) into (54) results in

$$
\dot{V} \leq-c V+\lambda
$$

where the positive constant $c$ and nonnegative constant $\lambda$ are given by

$$
c=\left(\frac{\min \left(k_{11}, k_{21}, k_{31}, k_{41}, \gamma_{1}, \gamma_{2}\right)}{\max \left(1, \delta_{1}, \delta_{2}\right)}\right), \quad \lambda=\sup _{t \geq 0}\left(\frac{\delta_{1}}{4 \epsilon_{1}} \dot{w}^{2}(t)+\frac{\delta_{2}}{4 \epsilon_{3}} \dot{\Delta}^{2}(t)\right)
$$

where for simplicity of presentation, we have used only $t$ in the argument of $\dot{w}$ and $\dot{\Delta}$. Solving the differential inequality (56) shows that $V$ exponentially converges to a ball centered at the origin with the radius $R_{V}=\lambda / c$. This implies that the closed loop system consisting of (16)), (20), (24) and (28) is forward complete. Moreover, convergence of $V$ implies that all the errors

$x_{i e}, i=1, \ldots, 4$, and the disturbance observer errors $w_{e}$ and $\Delta_{e}$ exponentially converge to a ball centered at the origin with a radius $R_{e}=\sqrt{\frac{\lambda}{c \min \left(1, \delta_{1}, \delta_{2}\right)}}$. Moreover, in the case where the derivatives of the disturbances $w$ and $\Delta$ can be ignored, we have $\lambda=0$. This means that all the state errors $x_{i e}, i=1, \ldots, 4$, and the disturbance observer errors $w_{e}$ and $\Delta_{e}$ exponentially converge zero.

\section{References}

Aker, M. H. (1998). Active Heave Compensator. Technical Bulletin, Kristiansand. Norway.

Do, K. D. (2007). Bounded controllers for formation stabilization of mobile agents with limited sensing ranges. IEEE Transactions on Automatic Control 52(3), 569-576.

Do, K.D., Z.P. Jiang and J. Pan (2002). Universal controllers for stabilization and tracking of underactuated ships. Systems and Control Letters 47, 299-317.

Fossen, T. I. (1994). Guidance and Control of Ocean Vehicles. John Willey and Sons. New York.

Fossen, T. I. and J. P. Strand (2001). Nonlinear passive weather optimal positioning control (wopc) system for ships and rigs: Experimental results. Automatica 37, 701-715.

Grimble, M. J., R. J. Patton and D. A. Wise (1980). The design of dynamic positioning control systems using stochastic optimal control theory. Optimal Contr. Applicat. Methods 1, 167202.

Johansen, T.A., T. I. Fossen, S. I. Sagatun and F.G. Nielsen (2003). Wave synchronizing crane control during water entry in offshore moonpool operations - experimental results. IEEE Journal of Oceanic Engineering 28, 720 - 728.

Khalil, H. (2002). Nonlinear systems. Prentice Hall.

Korde, U. A. (1998). Active heave compensation on drill-ships in irregular waves. Ocean Engineering 25(7), 541-561.

Krstic, M., I. Kanellakopoulos and P.V. Kokotovic (1995). Nonlinear and adaptive control design. Wiley. New York. 
Lloyd, A.R.J.M. (1998). Seakeeping Ship Behaviour in Rough Weather. Ellis Horwood Ltd.

Marconi, L., A. Isidori and A. Serrani (2002). Autonomous vertical landing on an oscillating platform: an internal-model based approach. Automatica 38, 21-32.

Merritt, H. E. (1967). Hydraulic Control Systems. Wiley. New York.

Sarpkaya, T. (1981). Mechanics of wave forces on offshore structures. Van Nostrand Reinhold Co. New York.

Skaare, B. and O. Egeland (2006). Parallel force/position crane control in marine operations. IEEE Journal of Oceanic Engineering 31, 599 - 613.

Soresen, A. J. (2005). Structural issues in the design and operation of marine control systems. Annual Reviews in Control 29, 125-149.

Teel, A. R. (1992). Global stabilization and restricted tracking for multiple integrators with bounded control. Systems and Control Letters 18, $165-171$.

Yao, B., F. Bu and G. T. C. Chiu (2001). Non-linear adaptive robust control of electro-hydraulic systems driven by double-rod actuators. International Journal of Control 74(8), 761-775. 